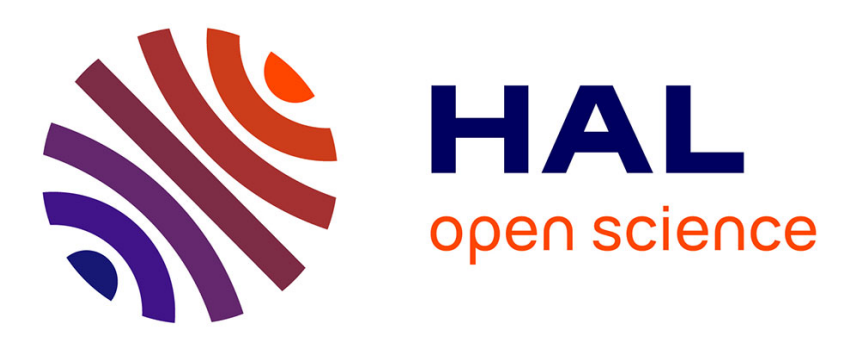

\title{
Les milieux d'affaires florentins, le commerce des draps et les marchés ottomans à la fin du XVe et au début du XVIe siècle
}

\author{
Ingrid Houssaye Michienzi
}

\section{- To cite this version:}

Ingrid Houssaye Michienzi. Les milieux d'affaires florentins, le commerce des draps et les marchés ottomans à la fin du XVe et au début du XVIe siècle. Mélanges de l'École française de Rome - Moyen Âge, 2015, Italians and Eastern Europe in Late Middle Ages / Les Italiens et l'Europe orientale au Bas Moyen Âge / Italiani ed Europa orientale nel basso Medioevo, 127/2, 10.4000/mefrm.2753 . hal-02386181

\section{HAL Id: hal-02386181 \\ https://hal.science/hal-02386181}

Submitted on 15 Sep 2021

HAL is a multi-disciplinary open access archive for the deposit and dissemination of scientific research documents, whether they are published or not. The documents may come from teaching and research institutions in France or abroad, or from public or private research centers.
L'archive ouverte pluridisciplinaire $\mathbf{H A L}$, est destinée au dépôt et à la diffusion de documents scientifiques de niveau recherche, publiés ou non, émanant des établissements d'enseignement et de recherche français ou étrangers, des laboratoires publics ou privés. 
Mélanges

de l'École française de Rome

Moyen Âge
Mélanges de l'École

française de Rome - Moyen

Âge

127-2 (2015)

Italians and Eastern Europe in Late Middle Ages / Les Italiens et l'Europe orientale au Bas Moyen Âge / Italiani ed Europa orientale nel basso Medioevo - Varia - Ateliers doctoraux - Regards croisés

Ingrid Houssaye Michienzi

\section{Les milieux d'affaires florentins, le commerce des draps et les marchés ottomans à la fin du $X V^{\mathbf{e}}$ et au début du $X V I^{e}$ siècle}

\begin{abstract}
Avertissement
Le contenu de ce site relève de la législation française sur la propriété intellectuelle et est la propriété exclusive de l'éditeur.

Les œuvres figurant sur ce site peuvent être consultées et reproduites sur un support papier ou numérique sous réserve qu'elles soient strictement réservées à un usage soit personnel, soit scientifique ou pédagogique excluant toute exploitation commerciale. La reproduction devra obligatoirement mentionner l'éditeur, le nom de la revue, l'auteur et la référence du document.

Toute autre reproduction est interdite sauf accord préalable de l'éditeur, en dehors des cas prévus par la législation en vigueur en France.
\end{abstract}

\section{revues.org}

Revues.org est un portail de revues en sciences humaines et sociales développé par le Cléo, Centre pour l'édition électronique ouverte (CNRS, EHESS, UP, UAPV).

\section{Référence électronique}

Ingrid Houssaye Michienzi, «Les milieux d'affaires florentins, le commerce des draps et les marchés ottomans à la fin du XV et au début du XVI siècle », Mélanges de l'École française de Rome - Moyen Âge [En ligne], 127-2 | 2015, mis en ligne le 14 décembre 2015, consulté le 14 décembre 2015. URL : http://mefrm.revues.org/2753

\section{Éditeur : École française de Rome \\ http://mefrm.revues.org}

http://www.revues.org

Document accessible en ligne sur :

http://mefrm.revues.org/2753

Document généré automatiquement le 14 décembre 2015

(c) École française de Rome 


\section{Les milieux d'affaires florentins, le commerce des draps et les marchés ottomans à la fin $\mathrm{du} X \mathrm{~V}^{\mathrm{e}}$ et au début du $\mathrm{XVI}^{\mathrm{e}}$ siècle}

Les activités des négociants florentins, grâce à d'importants dépôts d'archives sur lesquels se sont penchées plusieurs générations d'historiens, sont relativement bien connues, mais il est des lacunes historiographiques persistantes qui nécessitent d'être comblées. Peu d'études ont mis en relation Florence, ses marchands et les pays d'Islam ${ }^{1}$. Ces espaces, souvent considérés comme marginaux, sont le plus souvent balayés en quelques lignes et se perdent dans la masse d'écrits concernant l'histoire de la ville et de son expansion, ou dans celle concernant d'autres cités marchandes italiennes. Pour Venise, Gênes, Pise, ou encore quelques villes moins importantes de l'espace adriatique comme Ancône ou Raguse (Dubrovnik), ce champ d'étude a bénéficié d'un intérêt constant. Or pour l'histoire florentine il s'agit d'un objet nouveau, qui exige d'avoir recours à des documents jusqu'à maintenant délaissés et à des questionnements renouvelés. Ainsi, si les relations commerciales de Venise ou de Gênes avec l'Empire ottoman ont fait l'objet de nombreux écrits, on ne peut en dire autant de Florence et de ses marchands. D'aucuns penseront que le sujet est inexistant et que la présence florentine sur les marchés ottomans était trop faible pour avoir laissé des traces importantes.

Il est vrai que les négociants florentins, puis leurs navires, voguèrent plus tardivement en Méditerranée orientale. Leur attention était davantage tournée vers l'Occident méditerranéen, même s'il existait déjà depuis plusieurs siècles des noyaux de marchands florentins dans les plus importantes places commerciales de Méditerranée orientale. Une localisation tyrrhénienne des ports utilisés par les Florentins engageait davantage les marchands vers le commerce avec l'Occident européen et africain, mais il ne faut pas oublier que les Barcelonais, bien plus à l'Ouest fréquentaient abondamment et depuis déjà longtemps les ports orientaux ${ }^{2}$. La suprématie vénitienne en Méditerranée orientale put largement freiner leurs ambitions, mais ce sont davantage les liens étroits tissés entre Florence et Pise, qui déterminèrent l'orientation géographique de la stratégie florentine. En effet, avant de posséder ses propres moyens de navigation et de mettre en place une politique navale à partir de $1421^{3}$, c'est essentiellement sous couvert pisan que les marchands florentins négociaient en Méditerranée. Il est donc logique qu'une fois autonome au niveau maritime, Florence ne fasse que prolonger des contacts et des relations élaborées auparavant par ses ressortissants. Néanmoins, un changement s'opéra dans le premier tiers du $\mathrm{XV}^{\mathrm{e}}$ siècle. L'envoi d'une flotte commerciale florentine démontre alors l'intérêt naissant et croissant de la République pour une expansion commerciale en Méditerranée orientale 4 .

Dès la conquête de Pise en 1406, les Florentins essayèrent de récupérer son héritage à Constantinople, notamment ses privilèges et l'église dont les Pisans jouissaient. Ce fut sans succès en 1416 auprès de Manuel II Paléologue, et de nouveau en 1430 et en 1436 auprès de Jean VIII Paléologue. Ce n'est qu'en 1439, à l'occasion du concile de Florence ${ }^{5}$, c'est-àdire près de 33 ans plus tard, que l'empereur Jean VIII Paléologue transféra à la République de Florence les droits que les Pisans possédaient à Constantinople ainsi que l'église de Saint Pierre et leur maison commune ${ }^{6}$. Le décret accordé reconnaissait à Florence le droit de nommer des consuls pour administrer ses ressortissants et fixait les droits de douane. Ceci marqua la véritable installation d'un groupe régulier de marchands florentins à Constantinople. Auparavant, ils devaient s'abriter sous pavillon catalan. En effet, en 1437, afin de faire enregistrer en justice un acte à travers lequel ils apparaissaient en tant qu'acheteurs dans un marché d'alun, les marchands florentins avaient été reçus dans la maison des Catalans par 
le chancelier du consulat « catalan-florentin ${ }^{7}$. Ce décret fut également le coup d'envoi de l'établissement de liaisons maritimes régulières entre les deux cités, ce qui n'exclut pas que des embarcations florentines voguaient déjà vers Constantinople avant cette date. En 1437, un registre de ricordanze de la bottega d'arte della lana d'Alamanno di Iacopo Salviati et Niccolò di Sandro del Barbigia mentionne par exemple une vente de vingt draps de laine, expédiés sur une galée florentine conduite par Francesco Mannelli, et destinés à être vendus à Constantinople ou dans un autre lieu de Romanie :

Luigi di Franciescho Lioni de' dare a dì IIII ${ }^{\circ}$ di marzo $1437 \mathrm{~F}$. novecie(n)to d(anari) ; sono $\mathrm{p}(\mathrm{er})$ panni XX cioè : $\mathrm{X}$ turchini a dritto, e X v(er)di de' secho(n)di ; i q(u)ali gli mandamo $\mathrm{i}(\mathrm{n})$ acchomanda $\mathrm{i}(\mathrm{n})$ sino a dì V d'aghosto $1437 \mathrm{p}(\mathrm{er})$ la ghalea fiorentina padroneggiata $\mathrm{p}(\mathrm{er})$ Franciescho Mannelli, p(er) finirgli i(n) Ghosta(n)tinopoli o nelle parti di Romania ${ }^{8}$.

La conquête turque de 1453 changea la donne. L'État florentin, tout comme la République de Venise, celle de Gênes ou bien les souverains napolitains, dut maintenir un équilibre délicat entre ses intérêts commerciaux et le sentiment de croisade ${ }^{9}$. Néanmoins, les intérêts commerciaux supplantèrent rapidement l'idée de croisade ${ }^{10}$. Florence, comme les autres pouvoirs latins, devint un partenaire commercial de l'Empire ottoman et n'était pas encline à encourager la guerre contre les Turcs. En effet, à la différence de Venise ou de Gênes, elle n'était pas directement menacée par l'expansion ottomane puisqu'elle n'avait aucune possession territoriale en Méditerranée orientale. Défendre la croisade aurait ainsi bénéficié à Venise, son concurrent majeur en Méditerranée orientale. Si au début, la cité-État contribua sans enthousiasme aux efforts de croisade à travers la perception de décimes collectées dans son territoire, son attitude changea à la suite de l'obtention de privilèges et de protection du sultan Mehmed II ${ }^{11}$. Ce dernier confirma en effet les privilèges de Florence et la ligne de navigation reprit, passant de l'envoi d'une seule galée à un convoi de deux galées puis parfois de trois, signe d'un accroissement conséquent de son commerce en ces terres. Les privilèges furent régulièrement renouvelés par les différents sultans, et des consuls se succédèrent à la tête de la communauté florentine. Cependant, en dépit des avantages dont la cité jouissait, la position des Florentins sur le territoire ottoman n'était pas bien établie. La situation changea légèrement à la faveur des conflits entre Venise et l'Empire, principalement entre 1463 et 1479 puis entre 1499 et $1503^{12}$. Les longues guerres, qui marquèrent la fin du règne de Mehmed II et le début de celui de son fils Bajazet II, donnèrent aux sultans l'occasion de favoriser Florence afin de briser leur dépendance de Venise, ce qui n'arrangea bien entendu pas les relations entre la Sérénissime et la cité du Lys ${ }^{13}$. Florence reçut des faveurs qui contribuèrent considérablement à l'accroissement de son commerce sur les marchés ottomans ${ }^{14}$.

Ainsi, les lacunes historiographiques concernant les relations commerciales entre Florence et l'Empire ottoman ne sont pas liées à l'insignifiance de ces dernières, mais sont davantage le résultat d'une documentation peu abondante et dispersée. Jusqu'à maintenant, trois sources ont été utilisées pour étudier les Florentins et leur commerce dans l'Empire ottoman aux $\mathrm{XV}^{\mathrm{e}}$ et $\mathrm{XVI}^{\mathrm{e}}$ siècles : les documents de nature officielle conservés principalement dans les archives de Florence recueillis et édités par Giuseppe Müller en $1879^{15}$, les « morceaux choisis » de la correspondance de Giovanni Maringhi traduits en anglais par Gertrude R.B. Richards et publiés en $1932^{16}$, et la chronique du Florentin Benedetto Dei - partiale et anti vénitienne rédigée dans la seconde moitié du XV $\mathrm{XV}^{\mathrm{e}}$ siècle et éditée en $1985^{17}$. Les rares études sur le sujet dérivent quasiment toutes de ces trois ouvrages, à l'exception des articles d'Hidetoshi Hoshino, notamment centrés sur l'examen d'un livre de compte tenu à Brousse et conservé à Florence ${ }^{18}$ et de Bruno Dini à partir notamment des contrats d'assurance du registre de Raggio di Nofero Raggi ${ }^{19}$.

7 L'étude ici proposée cherche à la fois à combler ce vide historiographique et à bouleverser l'image classique d'une Méditerranée orientale dominée par Gênes et Venise et leurs constantes rivalités. Le corpus réuni, composé de documents issus de la pratique des affaires - registres de comptes et correspondances - offre d'abondantes informations sur le commerce qui était alors effectué dans l'Empire par les marchands florentins et permet d'analyser leurs 
mécanismes d'insertion sur les plus importants marchés de l'Empire ottoman à la fin du $X^{2}$ et au début du XVI $\mathrm{Xiècle}^{20}$. L'analyse de cette documentation pose les fondations d'une féconde étude sur les milieux d'affaires florentins et l'Empire ottoman au $\mathrm{XV}^{\mathrm{e}}$ et $\mathrm{XVI}^{\mathrm{e}}$ siècles en proposant à la fois des pistes de réflexion et les premiers résultats d'une vaste et prometteuse enquête.

\section{Les draps de laine florentins, clefs de l'insertion sur les marchés ottomans}

Perdus au sein de l'immensité du fonds Salviati ${ }^{21}$, trois registres de comptes rédigés à Constantinople, et plus précisément à Pera ${ }^{22}$, entre 1491 et 1493 sont d'une grande richesse pour tous ceux qui s'intéressent aux activités des marchands florentins dans l'espace méditerranéen. Ces registres sont donc au nombre de trois. Dans le premier, intitulé Giornale e Ricordi, Giovanni di Marco Salviati écrit ses activités quotidiennes du 31 octobre 1491 au 12 août 1493, ainsi que « les choses dont il faut se souvenir » (Ricordi) comme des copies d'assurance ou de chargements de navire. Les informations qu'il contient sont ensuite reportées dans un livre analytique des Debitori e Creditori contenant les comptes de personnes, de marchandises, de frais et de gestion. Les deux premiers livres doivent ainsi être lus parallèlement. Le troisième registre est également de cette nature mais il ne fonctionne pas avec les deux autres. Il est tenu du 22 mars au 7 août 1493 et se focalise sur les envois de produits textiles de Florence, et notamment sur l'exportation de draps de laine. Giovanni Salviati tint ses registres jusqu'à sa mort qui survint en novembre 1493. En effet, une lettre adjointe à l'un des registres, datée du 16 novembre 1493, nous informe qu'il était alors très malade de la peste ${ }^{23}$. Il décéda certainement quelques jours plus tard. Les livres de comptes sont ensuite tenus par Antonio Miniati qui géra l'héritage de Giovanni Salviati depuis Florence ${ }^{24}$. Ils furent ainsi certainement rapatriés à Florence avec les effets personnels de Giovanni Salviati. Ces documents sont très importants puisqu'à ce jour ils comptent parmi le peu de registres comptables tenus par des Florentins à Constantinople, à l'époque ottomane, que nous connaissions.

L'analyse des opérations contenues dans ces registres permet de caractériser le commerce qui était réalisé par Giovanni Salviati et d'autres marchands florentins qui étaient en rapport avec lui au sein des marchés ottomans. Les draps de laine consistaient l'essentiel des exportations florentines auxquelles s'ajoutaient des tissus de soie. Giovanni Salviati enregistra dans ses livres de compte, sur une durée de 21 mois, la vente de 310 tissus de laine de différents types (figure 1). Les échanges étaient effectués en aspres et c'est en cette monnaie locale que Giovanni Salviati tint sa comptabilité, à quelques exceptions près. Il en livra à plusieurs reprises l'équivalence avec le ducat d'or de Venise. Ainsi, il écrivit le 7 juillet 1492 que le ducat d'or vénitien valait 52 aspres $^{25}$. Dix jours plus tard, il indiquait que 63 ducats d'or donnaient 3276 aspres $^{26}$, ce qui confirme la valeur donnée précédemment.

Tab. 1 - Types de tissus vendus par Giovanni Salviati dans l'Empire ottoman du 31 octobre 1491 au 30 juillet 1493

\begin{tabular}{|l|l|l|l|}
\hline $\begin{array}{l}\text { Type de tissus importés sur } \\
\text { les marchés ottomans }\end{array}$ & Nombre de pièces & $\begin{array}{l}\text { Prix unitaire moyen(en } \\
\text { aspres) }\end{array}$ & Montanttotal(en aspres) \\
\hline Panni sopramani & 127 & 1453 & 184475 \\
\hline Panni di garbo & 51 & 1303 & 66470 \\
\hline $\begin{array}{l}\text { Panni di garbo bagnati e } \\
\text { cimati }\end{array}$ & 48 & 1210 & 58120 \\
\hline Panni bastardi & 32 & 1300 & 41600 \\
\hline Panni corsivi & 17 & 1246 & 21190 \\
\hline $\begin{array}{l}\text { Panni corsivi bagnati e } \\
\text { cimati }\end{array}$ & 8 & 1160 & 9280 \\
\hline Panni sanmartini & 7 & 2619 & 18336 \\
\hline Panni (sans précision) & 20 & 1404 & 28080 \\
\hline TOTAL & 310 & & 427551 \\
\hline
\end{tabular}


Les draps de laine étaient issus des ateliers florentins, et nous disposons pour beaucoup d'entre eux du nom du fabricant. Quelques tissus de luxe (dits sanmartini ${ }^{27}$ ), réalisés à partir de laine anglaise chère et de haute qualité, étaient commercialisés, mais les échanges concernaient massivement des tissus de qualité inférieure réalisés à partir de laines autres que de la laine anglaise provenant des pourtours du Bassin méditerranéen occidental, dits panni di garbo ${ }^{28}$. Ces derniers apparaissent dans la documentation sous diverses appellations : panni di garbo, bastardi, sopramani, corsivi. Les bastardi étaient des tissus qui n'étaient pas entièrement teints et qui laissaient en blanc une certaine quantité de laine. Les sopramani étaient réalisés à partir d'une laine castillane, appelée lana sopramano, de bonne qualité, qui se différenciait ainsi de toutes les autres laines d'origine ibérique de qualité inférieure comme la laine de San Matteo. Importée de manière conséquente à partir de la fin du $\mathrm{XV}^{\mathrm{e}}$ siècle à Florence, elle permit l'amélioration des panni di garbo. Ces panni sopramani dominaient les échanges avec l'Empire ottoman. Enfin, la dénomination de panni corsivi renvoie à une expression utilisée dans la documentation se référant à une unique mesure standard de produits différents ${ }^{29}$. D'après la Pratica di mercatura de Pegolotti, les Florentins jouissaient déjà au début du $\mathrm{XIV}^{\mathrm{e}}$ siècle d'une réduction des droits de douane dans l'Empire byzantin. Il constatait déjà l'importance des panni fiorentini à Constantinople, en Morée et dans les îles de la Grèce ${ }^{30}$. Si leur présence numérique n'était pas très importante, le commerce avec ces contrées passait par d'autres intermédiaires, et notamment Venise, ce que ne manquait pas de rappeler le doge de la cité, Tommaso Mocenigo, sur son lit de mort, lorsqu'il songeait aux immenses richesses de sa ville :

\begin{abstract}
Voi avete veduto come i Fiorentini mettono ogni anno panni 16000 finissimi, fini e mezzani in questa terra, e noi gli mettiamo nell'Apulia, pel reame di Sicilia, per la Barberìa, in Sorìa, in Cipre, in Rodi, per l'Egitto, per la Romania, in Candia, per la Morea, per l'Istria. E ogni settimana i detti Fiorentini conducono qui ducati di tutte le sorte 7 000, che sono ducati 392000 all'anno. Comperano lane francesche, catalanesche, cremisi e grane, sete, ori, argenti filati, cere, zuccheri e gioie, con benefizio della nostra terra. Così tutte le nazioni fanno a questo modo. Però vogliate conservarvi nel modo in cui vi trovate, che sarete superiori di tutti. Il signor Iddio vi lasci conservare, reggere e governare in bene ${ }^{31}$.
\end{abstract}

12 La mise sur pied d'une politique navale et d'une expansion maritime à partir de 1421 fut pour Benedetto Dei la cause majeure de la haine des Vénitiens contre les Florentins, ce que confirment les discours du doge Mocenigo $^{32}$. Benedetto Dei rapporte dans sa chronique des quantités de draps de laine envoyés vers l'Empire ottoman qui, recoupées avec d'autres références, semblent crédibles. Ainsi, en 1470, 8000 draps de laine furent exportés dans l'Empire, 7500 en 1471 et 8000 en 1472. En 1474, malgré la peste à Constantinople, les exportations de draps de laine se montèrent à 3300 pièces auxquelles s'ajouta un montant de 60000 florins de tissus de soie vendus au sultan, puis stagnèrent à 3000 pièces en $1476^{33}$. Quelques années plus tard, en 1483, les accords conclus avec le sultan Bajazet II autorisaient les Florentins à importer jusqu'à 5000 pièces de tissus par an ce qui correspondait environ au tiers, voire à $40 \%$ de la production florentine de l'époque d'après les estimations réalisées par Hidetoshi Hoshino ou par Richard Goldthwaite ${ }^{34}$. Cette large possibilité d'importation souligne à la fois l'importance du marché turc pour l'industrie de la laine florentine et le succès que ces produits rencontraient dans l'Empire. L'existence d'un registre de compte de Giovanni Salviati spécialement dédié aux draps de laine, et aux tissus de soie dans une moindre mesure, importés dans l'Empire illustre aussi l'enjeu de ce marché qui conduisit à la création à Florence d'une agence dédiée au contrôle des relations commerciales avec les Turcs : les Procuratori delle cose di Romania $a^{35}$.

13 L'importante quantité d'exportation de draps de laine florentins vers Constantinople compta pour beaucoup dans la reprise de l'Arte della Lana et dans son orientation vers la production de draps de garbo, ce que l'historien japonais Hidetoshi Hoshino qualifia de «garbizzazione » de la manufacture lainière de la cité, c'est-à-dire le déplacement vers une production de tissus de laine de qualité moyenne ${ }^{36}$. L'analyse de registres du fonds Salviati est très évocatrice du changement de cap de la production florentine. Le fonds Salviati de la Scuola Normale di Pisa conserve près de cent ans de comptabilités de botteghe dell'Arte della lana quasiment 
interrompues, de la fin du XIV siècle au premier tiers du XVI ${ }^{\mathrm{e}}$ siècle. Si dans les premiers registres, les draps produits par les ateliers sont de type San Martino, la situation changea dans les années 1480. Les Salviati semblaient alors posséder plusieurs entreprises dont deux étaient dédiées au travail des draps de laine : l'une de type San Martino, l'autre de type garbo. Le grand livre .BB. tenu à partir de $1486^{37}$ mentionne, à côté des lanaioli Giuliano di Francesco et Iacopo di Giovanni Salviati, spécialisés dans le drap de type San Martino, la bottega de la via del Palagio, «nostri lanaioli in garbo ».

Marché de consommation intéressé par les beaux tissus florentins, l'Empire ottoman constituait un partenaire économique de premier choix pour les négociants florentins et pour le commerce de leurs draps, notamment de qualité secondaire. Un legaggio, c'est-àdire un inventaire complet de marchandises expédiées, conservé dans un des registres de Constantinople, fait état de l'expédition depuis Florence de 40 ballots contenant chacun quatre draps de laine ${ }^{38}$, avec des précisions quant au type de drap, au fabricant, à ses dimensions et à sa couleur. D'après le tableau récapitulatif ci-dessous (figure 2), $85 \%$ de ces draps, produits à Florence dans différents ateliers, étaient fabriqués avec des laines moins onéreuses que la laine anglaise, dont $40 \%$ avec de la bonne laine castillane. Seuls $15 \%$ d'entre eux constituaient encore une marchandise de luxe.

Tab. 2 - Tissus contenus dans une liste de $\mathbf{4 0}$ ballots expédiés de Florence vers Constantinople en 1493.

\begin{tabular}{|l|l|l|l|l|}
\hline & $\begin{array}{l}\text { Numérotation des } \\
\text { ballots }\end{array}$ & Nombre de ballots & Nombre de draps & $\begin{array}{l}\text { Proportion de } \\
\text { l'échantillon }\end{array}$ \\
\hline Panni bastardi & de 1 à 10 & 10 & 40 & $25 \%$ \\
\hline Panni sopramani & de 11 à 26 & 16 & 64 & $40 \%$ \\
\hline Panni corsivi & de 27 à 34 & 8 & 32 & $20 \%$ \\
\hline Panni sanmartini & de 35 à 40 & 6 & 24 & $15 \%$ \\
\hline TOTAL & & 40 & 160 & 100 \\
\hline
\end{tabular}

D'après la documentation conservée dans le fonds Salviati, il s'avère que les draps produits par les ateliers florentins, ou achetés en vue de leur commercialisation ${ }^{39}$, alimentaient à partir des années 1480, en plus du marché de Florence, un courant d'exportation qui augmenta sensiblement jusqu'à la deuxième décennie du XVI ${ }^{e}$ siècle. Trois directions principales le caractérisaient : le marché ottoman, Naples et l'Italie du Sud, et la péninsule Ibérique. De ces mêmes contrées étaient importées des matières premières nécessaires à l'industrie lainière et à celle de la soie : soie, laine et grana de péninsule Ibérique, soie du Levant et soie de Calabre. Ainsi, sur les marchés ottomans, les draps de laine servaient de monnaie d'échange en vue de se procurer des produits revendus à Florence à des artisans, notamment setaioli et lanaioli. Les provveditori de l'Arte della Lana affirmaient en effet en 1487 que le Levant était « in buona parte lo stomaco de' nostri panni garbi ${ }^{40} »$.

\section{Les mécanismes d'insertion sur les marchés ottomans}

En 1421, Florence acquit une façade maritime longtemps désirée, et commença à mettre sur pied une organisation de voyages de galées similaire à celle des mude vénitiennes ${ }^{41}$. Les premiers déplacements vers Constantinople eurent lieu dans les années 1430. Une ordonnance du Consul de la mer datant de 1447 en fixait les étapes : Modon, Négrepont, Gallipoli à l'aller et au retour, auxquelles s'ajoutaient Chio et Rhodes au retour. Ces deux dernières étapes, avec Candie et Modon faisaient également partie des itinéraires de galées se rendant à Alexandrie et à Beyrouth. Après la conquête turque de 1453, le service de navigation reprit. À la galée unique qui effectuait le voyage depuis la mise en place de la ligne s'ajouta une deuxième galée en $1457^{42}$, puis une troisième en $1461^{43}$. Les étapes réglementaires, fixées à nouveau en 1460 , étaient constituées de Chio et Gallipoli à l'aller, et de Gallipoli, Foglia et Chio au retour.

En raison des difficultés à trouver des armateurs pour effectuer les voyages en Romanie ou d'un contexte maritime trop périlleux, chaque année ne vit pas le départ des galées. De fait, un système parallèle était utilisé par les marchands peu soucieux d'attendre le départ du convoi officiel. Toutefois, les lignes florentines de navigation étaient abandonnées depuis près 
de quinze ans lorsque Giovanni Salviati était présent à Constantinople. Le dernier voyage eut lieu à la fin des années 1470. L'organisation parallèle qui s'était toujours maintenue fut redynamisée et fit la richesse des Ragusains et des Anconitains qui prenaient massivement en charge les marchandises florentines. Un des itinéraires dominant était effectué par voie de terre de Florence à Ancône, puis par mer d'Ancône à Raguse (Dubrovnik), pour se terminer à nouveau par voie terrestre jusqu'à Constantinople, voire Edirne (Andrinople) dans une moindre mesure. Cette route fut suivie par Bernardo Michelozzi et Bonsignore Bonsignori durant leur voyage au Levant en 1497-1498 ${ }^{44}$. Un deuxième itinéraire reliait Ancône et Constantinople par la mer. En juillet 1472, Florence ordonna au consul du Levant de ne pas exiger de gabelle des marchands florentins qui navigueraient sur des navires anconitains ${ }^{45}$. Néanmoins, ce parcours fut délaissé en raison de la progression des périls en Méditerranée. De fait, un troisième itinéraire fut emprunté, réduisant au minimum la traversée maritime : une longue route terrestre en péninsule italienne de Florence à Lecce suivi d'une courte traversée de Lecce à Valona ${ }^{46}$ (Vlora, Albanie), puis d'un parcours terrestre jusqu'à Constantinople. C'est le trajet que suivit Dimitri pour acheminer 42 ballots de draps de garbo et San Martino et quatre caisses de tissus de soie appartenant à la compagnie Alamanno et Iacopo Salviati et à celle de Priore et Strinato Strinati jusqu'à Pera. Dimitri, présenté dans les registres comme nostro garzone in Pera, devait certainement travailler pour le compte de Giovanni Salviati. Le 26 mars 1493, une copie d'un compte envoyé aux marchands florentins (Salviati et Strinati), conservée dans un des trois registres de Constantinople, fait état des frais de Dimitri qui voyagea jusqu'à Lecce chercher la marchandise, et fit l'itinéraire en sens inverse, de Lecce à Pera en effectuant plusieurs étapes ${ }^{47}$ : trajet maritime de San Cataldo (près de Lecce) à Valona $^{48}$, puis terrestre avec un convoi de 24 chevaux en passant par Kastoria (Macédoine ${ }^{49}$ et Edirne ${ }^{50}$ jusqu'à Constantinople ${ }^{51}$. La majorité des draps transitaient par Constantinople avant leur réexpédition vers d'autres marchés de l'Empire. D'après le registre d'assurances de Raggio di Nofero Raggi ${ }^{52}$, établies entre 1524 et 1526, près de $85 \%$ du commerce florentin avec le Levant concernait la capitale de l'Empire et les ports des Balkans, débouchés des voies terrestres.

Pour parfaire leur formation, des jeunes Florentins identifiés dans les registres de Giovanni Salviati, effectuaient également la navette entre Florence et Constantinople, en suivant différents itinéraires, afin de récupérer des sommes d'argent et d'acheminer des draps. Ainsi Antonio Biliotti se rendit à Florence pour récupérer la somme de 2500 aspres (en ducats d'or de Venise) auprès de Francesco Gherardi, débiteur de Giovanni Salviati ; il achemina en retour douze balles de tissus du même Francesco ${ }^{53}$. Lorenzo di Bartolomeo Bonagrazia, jeune homme au service des Serristori ${ }^{54}$, effectuait le même type de tâches ${ }^{55}$ : il se rendit à Florence auprès de Francesco Gherardi pour lui remettre la somme de 100 ducats « fra veneziani e unghari di peso ». Et Giramonte Ridolfi achemina des draps de laine de Florence ${ }^{56}$.

Les draps de laine issus des ateliers florentins pouvaient également être pris en charge à Florence pour être vendus par des tierces personnes sur les marchés ottomans. Le rôle des Ragusains et des Anconitains en tant qu'intermédiaires des Florentins s'accrut tout au long $\mathrm{du} \mathrm{XV}^{\mathrm{e}}$ siècle $\mathrm{e}^{57}$. Leur position heureuse sur les routes maritimes eut des effets conséquents sur le développement et le rôle maritime de ces deux villes. Ancône, débouché de l'Italie septentrionale et centrale, était politiquement dépendante du Saint-Siège ; Raguse se trouvait au point où les grandes routes terrestres provenant de l'intérieur de la Péninsule balkanique débouchaient sur la mer. Avec les succès ottomans du milieu du $X^{e}$ siècle, et l'adoption d'une attitude coopérative des Ragusains avec les sultans, le port et sa marine devinrent de plus en plus actifs et importants dans le commerce international ${ }^{58}$. La République jouissait de privilèges commerciaux dans les territoires ottomans, dont des droits de douane de $2 \%$, les plus bas pratiqués dans l'Empire ${ }^{59}$. À partir des années 1480, les registres de comptes des ateliers Salviati d'art de la laine contiennent davantage de comptes ouverts au nom de marchands de Raguse ou d'Ancône. Ainsi, entre le 19 mai 1487 et le $1^{\text {er }}$ avril 1489, Antonio di Luca di Bona, négociant ragusain, réalisa huit achats de draps de laine auprès de la compagnie Giuliano di Francesco et Iacopo di Giovanni Salviati, pour un montant de plus de 400 florins. 
Il s'agissait de draps produits par la bottega Salviati mais également de draps importés de Péninsule ibérique. En échange, et parfois sous forme de troc, le marchand ragusain vendit durant cette même période plus de 100 livres de soie levantine pour un montant d'environ 174 florins $^{60}$.

Des Anconitains avaient également des comptes ouverts dans des registres de comptes de cette même période, dont celui de Giovanni Salviati, qui avait par exemple enregistré une dette de 30 ducats de Venise contractée auprès de Francesco di Francesco di Gabriello d'Ancona ${ }^{61}$. Un grand nombre de compagnies florentines avaient des agents à Raguse, tel Martino Chiarini qui servit Iacopo Pazzi de 1448 à 1487. Le premier consul florentin y fut établi en $1495^{62}$. Et Giovanni Maringhi entretenait une correspondance en 1501 avec le consul florentin à Raguse, qui était alors Iacopo di Giuliano, et avec Nicolò Lippi, basé à Ancône. Ces derniers devaient accueillir un jeune Florentin chargé de fardeaux de soie à destination de Florence, nous rappelant ainsi le rôle de ces deux places dans l'itinéraire connectant la cité du Lys et les marchés ottomans ${ }^{63}$.

Des marchands florentins étaient néanmoins organisés sur les marchés levantins depuis le début du XV ${ }^{\mathrm{e}}$ siècle, et, comme nous l'avons vu, le sultan Mehmed II reconfirma les privilèges commerciaux des États italiens peu après la conquête de la cité byzantine. Comme les Génois et les Vénitiens, qui étaient toutefois présents dans la cité depuis le $\mathrm{X}^{\mathrm{e}}$ siècle et y étaient profondément enracinés ${ }^{64}$, Florence signa ses accords avec Mehmed II l'année de la conquête, et ses premiers bateaux y accostèrent en 1454, chargés de lainages. Le nombre de navires battant pavillon florentin se rendant à Constantinople augmentant progressivement, en 1461, un consul fut installé à Constantinople afin de préserver les intérêts des Florentins et superviser la communauté ${ }^{65}$. Durant les années 1461-1462 le Florentin Baldassare di Gualtieri Biliotti, correspondant et agent des Cambini, était établi à Pera où il agissait en accomandita avec Lorenzo di Ilarione Ilarioni, important lanaiolo de Florence. Ce dernier lui confia la somme importante de 6000 florins pour «traficare in mercatantia nelle parti di Levante e Romania e Turchia et in Constantinopoli e Pera et altri luoghi ». Le contrat, signé le 25 août 1462, devait durer trois ans et entrer en vigueur le $1^{\text {er }}$ septembre ${ }^{66}$.

En 1488, quelques années avant le séjour de Giovanni di Marco Salviati, Andrea di Bernardo de' Medici « ambasciatore a questo signore sultan Baiasid» se rendit à Constantinople afin de valider les accords octroyant de nouveaux statuts ${ }^{67}$. Le nombre de marchands florentins présents dans la cité et dans l'Empire s'accrut, ceux-ci s'établissant principalement à Constantinople, Edirne, Brousse et Gallipoli. En 1469, d'après les mémoires de Benedetto Dei, 51 Florentins étaient présents dans l'une de ces villes ${ }^{68}$. D'après Wilhelm Heyd, citant les Diarii du vénitien Marin Sanuto, les Florentins formaient un groupe de 60 à 70 marchands en 1507, dont les affaires atteignaient annuellement un chiffre de 500000 à 600000 ducats $^{69}$. Il s'agissait alors du groupe de marchands florentins le plus conséquent en terres étrangères. Dans les années 1460-1500, les Florentins établis à Pera et administrés par un consul (figure 3) - qui prit ensuite le titre d'émin à partir de 1500 dans la documentation - étaient ainsi devenu un groupe influent et prospère grâce à la politique menée par les sultans Mehmed II et son fils Bajazet II, et à la qualité de leur production textile.

En 1527, les privilèges octroyés aux Florentins par Mehmed II ${ }^{70}$, Bajazet II et Selim I $^{\text {er }}$ furent renouvelés par Soliman $\mathrm{I}^{\mathrm{er}}$; les garanties concernant la liberté de voyage et de commerce, les dispositions regardant la sécurité des biens et des personnes ainsi que les droits de douane équivalaient alors aux avantages octroyés aux Vénitiens en $1482^{71}$. Un Baylos supervisait la communauté, les affaires de ses ressortissants et avait juridiction sur les litiges entre Florentins. Les autres litiges étaient jugés par les cadis. Aucune responsabilité collective ni consulaire n'était invoquée. Chaque marchand était responsable de ses propres dettes et ne pouvait quitter le territoire ottoman sans les avoir réglées. Les Florentins étaient autorisés à circuler dans tout l'Empire. Ils étaient également libres de circuler sur la mer Noire, et ceux qui voyageaient sous pavillon florentin bénéficiaient des mêmes libertés une fois les taxes payées. À chaque arrivée de navire, les Florentins devaient offrir des cadeaux au sultan. Ceci fait bien entendu à des accords antérieurs renouvelés car à la date de 1527, Florence n'organisait plus de voyages de 
galées depuis bien longtemps. Les navires battant pavillon florentin étaient le fruit d'initiatives individuelles. Les Florentins avaient la permission d'amener avec eux des femmes esclaves qu'il fallait déclarer au cadi, et de louer les services de non-musulmans pour les servir et les assister, sans néanmoins pouvoir les emmener en Italie.

Deux éléments dans ces capitulations font référence aux itinéraires commerciaux des Florentins. Le premier concerne l'achat de soie sur le marché de Brousse puisqu'un article interdisait expressément aux collecteurs de faire payer un deuxième courtage aux Florentins à Constantinople pour la soie grège importée de Brousse par voie de terre. Giovanni Maringhi faisait ainsi voyager de la soie achetée à Brousse jusqu'à Constantinople, avant son expédition vers Florence : « L'aportatore di q(uest)a [lettera] sarà B(er)nardo Risaliti n(ost)ro, p(er) il quale vi si manda $\mathrm{f}$ (ardelli) 3 di seta leggi $[\ldots]$ che la chonperò in $\mathrm{B}(\mathrm{ur})$ sia el Ghazzeto $^{72}$ »

Le second mentionne un itinéraire commercial particulièrement prisé et laissant peu de place au trajet maritime. En effet, si les marchands florentins arrivaient à Valona, louaient un navire pour se rendre en Italie (à Lecce), se faisaient intercepter et séquestrer leurs biens par des Vénitiens, des Génois ou d'autres «infidèles », les autorités ottomanes demandaient à ce que les biens capturés leur soient restitués si les attaquants étaient trouvés sur des territoires ottomans. Cet article des capitulations évoque les dangers maritimes accrus en Méditerranée et l'itinéraire très terrestre qui, de fait, était le plus utilisé par les Florentins. Déjà, dans une lettre du 8 janvier 1499, Florence remerciait le Capitaine général de la mer à Valona de ses bonnes dispositions à l'égard des marchands florentins. Puis en mai 1504, Mustafa, sandjakbey de Valona ${ }^{73}$, invita les Florentins en Albanie, avec leurs marchandises, leur promettant des avantages ; cette invitation se conclut par l'élection de Bernardo Rucellai en tant qu'émin de Valona en décembre $1505^{74}$.

Tab. 3 - Ambassadeurs et consuls florentins à Constantinople de 1453 aux années $1530^{75}$

\begin{tabular}{|c|c|c|}
\hline Année & Nom & Fonction / attributions \\
\hline 1461 & Mainardo Ubaldini & $\begin{array}{l}\text { Consul des Florentins (d'après } \\
\text { Benedetto Dei) }\end{array}$ \\
\hline 1466 & Giovanni Marsupini & $\begin{array}{l}\text { Citoyen florentin et anconitain, } \\
\text { consul des Florentins à Pera }\end{array}$ \\
\hline 1469 & Mainardo Ubaldini & $\begin{array}{l}\text { Consul florentin à PeraToujours en } \\
\text { exercice en } 1471\end{array}$ \\
\hline 1471 & Bartolomeo del Nero & $\begin{array}{l}\text { Consul du LevantElu le } 26 \text { octobre } \\
1471\end{array}$ \\
\hline 1472 & Carlo Baroncelli & $\begin{array}{l}\text { Consul du LevantElu en septembre } \\
1472 \text { Nomination d'un vice-consul en } \\
1476 \text { en raison son retour en Italie }\end{array}$ \\
\hline 1477 & Lorenzo Carducci & Consul florentin à Constantinople \\
\hline 1479 & Antonio de' Medici & $\begin{array}{l}\text { Ambassadeur auprès du sultan } \\
\text { Mehmed II chargé d'obtenir } \\
\text { l'extradition de Bernardo } \\
\text { Bandini }{ }^{76} \text { Envoyé en juillet } 1479\end{array}$ \\
\hline 1481 & Leonardo Salvucci & $\begin{array}{l}\text { Consul florentin à PeraElu en janvier } \\
1481\end{array}$ \\
\hline 1488 & Andrea di Bernardo de' Medici & $\begin{array}{l}\text { Ambassadeur auprès du sultan } \\
\text { Bajazet IIEnvoyé en juin } 1488\end{array}$ \\
\hline 1492 & Leonardo di Benedetto Strozzi ${ }^{77}$ & $\begin{array}{l}\text { Consul florentin à } \\
\text { ConstantinopleEnvoyé en septembre } \\
1492\end{array}$ \\
\hline 1499 & Geri Risaliti & $\begin{array}{l}\text { Ambassadeur auprès du sultan } \\
\text { Bajazet II dans le but de « renovare } \\
\text { la antiqua amicitia »Envoyé en } \\
\text { février } 1499\end{array}$ \\
\hline $1500-1504$ & Andrea Carnesecchi & $\begin{array}{l}\text { Emin florentin à } \\
\text { ConstantinopleNommé en mai } 1500\end{array}$ \\
\hline $1504-1508$ & Pandolfo di Bernardo Corbinelli & $\begin{array}{l}\text { Emin florentin à } \\
\text { ConstantinopleNommé en mai } 1504\end{array}$ \\
\hline $1508-1512$ & Niccolò Morelli & $\begin{array}{l}\text { Emin florentin à } \\
\text { ConstantinopleNommé en mai } 1508\end{array}$ \\
\hline
\end{tabular}




\begin{tabular}{|l|l|l|}
\hline $1512-1515$ & Giuliano Lapi & $\begin{array}{l}\text { Emin florentin à } \\
\text { ConstantinopleNommé en mars 1512 }\end{array}$ \\
\hline 1513 & Francesco Antonio Nori & $\begin{array}{l}\text { Ambassadeur auprès du sultan } \\
\text { Sélim Ir dans le but de renouveler } \\
\text { les capitulations Capitulations } \\
\text { renouvelées en octobre 1499 }\end{array}$ \\
\hline 1515 & Zanobi de' Medici & $\begin{array}{l}\text { Emin florentin à } \\
\text { ConstantinopleNommé en février } \\
1515\end{array}$ \\
\hline 1524 & Giovanni Lotti & $\begin{array}{l}\text { Emin florentin à } \\
\text { ConstantinopleNommé en avril 1524 }\end{array}$ \\
\hline 1528 & Alessandro Sacchetti & $\begin{array}{l}\text { Emin florentin à } \\
\text { ConstantinopleNommé en avril 1528 }\end{array}$ \\
\hline 1528 & Piero di Banco degli Albizzi & $\begin{array}{l}\text { Ambassadeur auprès du sultan } \\
\text { Soliman pour obtenir du bléEnvoyé } \\
\text { en décembre 1528 }\end{array}$ \\
\hline
\end{tabular}

Giovanni Salviati résida à Pera certainement d'octobre 1491 à novembre 1493, mois où il trouva la mort. La lettre rédigée à Pera et datée du 16 novembre 1493 (figure 4), intégralement transcrite ci-après, donne quelques informations supplémentaires. Giovanni Salviati se trouvait alors au lit, très malade de la peste. Il reconnaissait à travers cet écrit une dette contractée auprès de Lorenzo Ridolfi, autre marchand florentin, dont le montant ne figurait pas dans ses registres de comptes. Pour faire foi à ses mots furent apportés le témoignage de plusieurs marchands florentins, dont Giovanni Maringhi, écrits de leur propre main. L'un d'entre eux, Carlo di Iacopo Baroncelli ${ }^{78}$, se trouvait dans une maison située en face de celle de Giovanni Salviati (di rimpetto) et dans laquelle résidait un autre négociant florentin, Alfieri Strinati. Il témoignait à travers la fenêtre de cette maison d'où il pouvait assister à toute la scène. Cette scène, d'un aspect assez sordide, informe à la fois du tissu urbain serré du quartier de Pera, mais également de la proximité de résidence de ces marchands, groupés certainement dans quelques rues. Alfieri Strinati était le principal partenaire commercial de Giovanni Salviati.

Tab. 4 - Scuola Normale Superiore di Pisa, Archivio Salviati, série I, registre 397, Constantinople, fol. XXIX, lettre écrite le 16 novembre 1493 à Pera.

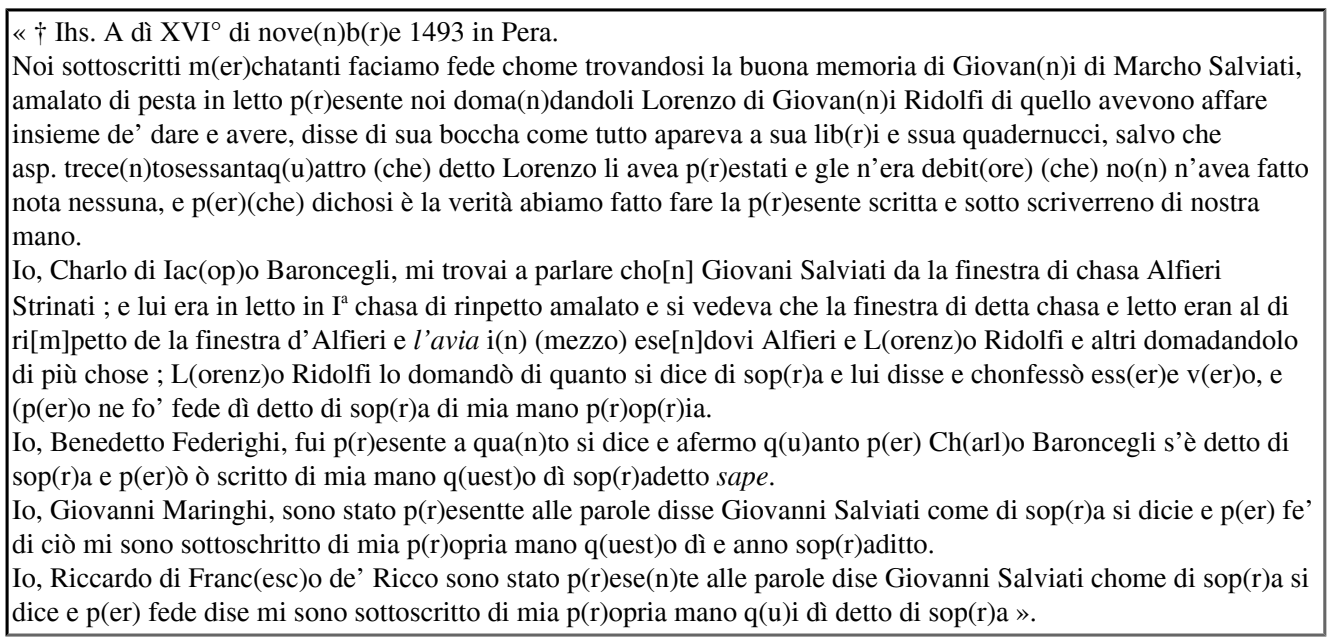

28 Giovanni Salviati possédait également un ou des entrepôts qu'il est impossible de situer géographiquement, dont il louait une partie à ce même Lorenzo Ridolfi qui avait également en location un entrepôt de savons ${ }^{79}$, un article dont nous retrouvons parfois la trace dans des livres de comptes florentins ${ }^{80}$.

29 Giovanni Salviati réalisa des déplacements durant ses deux années documentées de présence dans l'Empire, notamment à Edirne ${ }^{81}$ et à Brousse ${ }^{82}$. L'analyse de ses trois livres de comptes livre les noms de marchands et artisans florentins installés également à Constantinople tels Filippo Capponi et Andrea Berti, auxquels il prêta respectivement 500 et 40 aspres le 16 avril $1492^{83}$, Niccòlo di Giorgio, papetier florentin résidant à Venise mais alors à Pera ${ }^{84}$, en plus 
de Lorenzo di Giovanni Ridolfi, Giovanni Maringhi, Carlo di Iacopo Baroncelli, Riccardo di Francesco del Ricco ou Alfieri Strinati, déjà mentionnés. Le détail des nombreux comptes d'Alfieri Strinati permet d'entrevoir des dépenses liées non plus au grand commerce, mais à son usage personnel. La lettre transcrite ci-dessus évoquait une maison dans le quartier de Pera ; il payait également vingt aspres par mois à Leonardo Salucci, évoqué en tant que consul, pour la location d'une petite maison ${ }^{85}$, ainsi que 400 aspres annuels pour celle d'un jardin ${ }^{86}$, en plus de bois, de caisses de sucre, de confetti et de malvoisie (botte di malvagia) ${ }^{87}$. Sa clientèle, durant ses années d'exercice, était composée de personnes pour lesquelles nous n'avons pas toujours des informations détaillées. Elles sont enregistrées uniquement sous leur simple prénom ou leur prénom accompagné du nom de famille. Dans quelques cas figure le lieu de leur activité, ce qui permet d'avancer qu'il était en relations d'affaires avec des marchands installés à Constantinople, à Brousse, à Edirne et à Gallipoli. Enfin, pour certaines personnes est indiquée la religion dans le cas des juifs, ou bien l'appartenance ethnique pour les Grecs, les Turcs et les Vénitiens. Quelques conclusions peuvent néanmoins être avancées.

À Constantinople, ses principales relations étaient des marchands juifs qui tenaient boutique au Bedesten de Constantinople (bottegaio in Bisestano). Le Bedesten était le cœur économique de la cite ${ }^{88}$. Marché couvert, ce qu'on appelle aujourd'hui le Vieux Bazar, construit sous Mehmed II en 1456-1461, était le lieu de rencontre de marchands de diverses origines. Le mot bedesten provenait du terme persan bezestan, lui-même dérivant du mot bez signifiant vêtement ; il désignait de fait le «bazar des vendeurs de vêtements ». En 1473, il y avait cent vingt-quatre boutiques à l'intérieur du Bedesten et soixante-douze à l'extérieur. En 1519, les comptes du wakf indiquent qu'à cette date seul un tiers des boutiques étaient gérées par des non-musulmans ${ }^{89}$. Néanmoins, parmi les dix boutiquiers qui achetèrent des produits textiles à Giovanni Salviati (figure 5), seul l'un d'entre eux était turc ; les autres étaient tous juifs, alors que les registres du wakf indiquent la présence de seulement cinq marchands juifs tenant boutique dans le Bedesten en 1489, puis de 18 en 1520. À cette dernière date néanmoins, l'émigration massive de juifs d'origine ibérique vers l'Empire suite aux expulsions qui débutèrent en Espagne en 1492, vint gonfler le nombre de juifs détenteurs de boutiques.

Tab. 5 - Les boutiquiers au Bedesten de Constantinople d'après les registres de Giovanni Salviati, 1491-1493

\begin{tabular}{|l|l|l|l|l|}
\hline Acheteur & Origine & Marchandises & Quantité & Prix total(en aspres) \\
\hline \multirow{2}{*}{ Sabatino } & \multirow{2}{*}{ juif } & Panni sopramani & 8 & 12400 \\
\cline { 3 - 5 } & & Panni sopramani & 6 & 9240 \\
\hline \multirow{2}{*}{$\begin{array}{l}\text { Isache di Cordova } \\
\text { LUCuda e Sabatino }\end{array}$} & \multirow{3}{*}{ juifs } & Panni sopramani & 5 & 7575 \\
\hline & & Panni sopramani & 20 & 30750 \\
\cline { 2 - 5 } & $\begin{array}{l}\text { Panni garbi bagnati e } \\
\text { cimati }\end{array}$ & 4 & 4240 \\
\cline { 2 - 5 } & Panni sopramani & 5 & 7750 \\
\hline Elia MANSELLA & juif & Panni & 14 & 20370 \\
\hline \multirow{2}{*}{ Baba DAVIT } & \multirow{2}{*}{ juif } & Panni samartini & 8 & 18336 \\
\hline & Panni sopramani & 12 & 18960 \\
\hline Begliamino & juif & Panni bastardi & 8 & 10400 \\
\hline $\begin{array}{l}\text { Mordechai e Abram } \\
\text { (comp.) }\end{array}$ & \multirow{2}{*}{ juifs } & $\begin{array}{l}\text { Panni corsivi bagnati } \\
\text { e cimati }\end{array}$ & 8 & 9280 \\
\hline Maomet & turc & Panni corsivi & 5 & 5950 \\
\hline
\end{tabular}

À Constantinople, la présence de la cour ottomane, consommatrice de biens de luxe, représentait également une source importance de revenus pour les marchands européens ${ }^{90}$. Entre le 26 juin et le ${ }^{\text {er }}$ juillet 1493, Giovanni Salviati vendit 14 tissus de laine de type garbo et 50 de type sopramano à la Sublime Porte (la Porta del Signore), ainsi qu' un brocart écarlate, le tout pour un montant de 122210 aspres, soit environ 2350 ducats vénitiens. L'opération ci-dessous concerne la vente de neuf draps de laine à la Porte (fig. 1). 
Fig. 1 - Vente de tissus à la Sublime Porte, le 26 juin $1493^{91}$

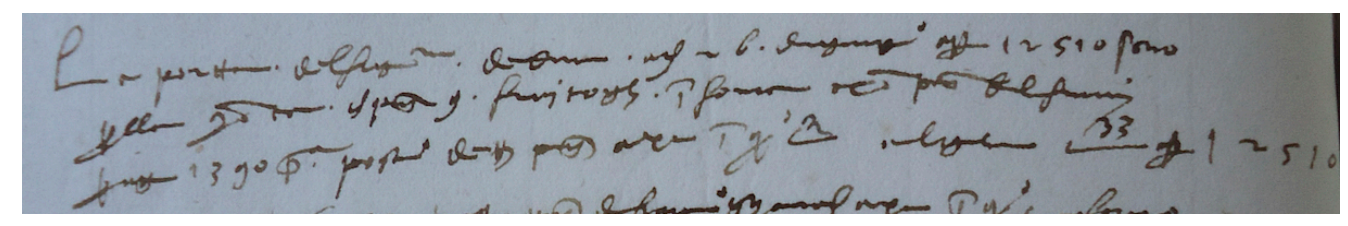

La Portta del Sig(no)re de' dare, a dì 26 di giug(n)o asp. 12510 ; sono p(er) lla mo(n)ta di pan(ni) 9 servitogli i(n) soma cho(n) pan(ni) d'Alfieri, p(er) asp. 1390 p(ezz)a ; posto detti pan(ni) av(er)e i(n) q(uest)o c.3, al g[iorna]le c.33 -------------------- asp. 12510

D'autres marchés, assez distants de Constantinople, attiraient également les négociants florentins. Brousse (nommée Bursa dans la documentation), capitale des Ottomans de 1326 à 1402, était la plateforme du réseau commercial anatolien. Les marchands musulmans y contrôlaient les caravanes en provenance d'Iran, transportant des marchandises légères mais au prix élevé (produits tinctoriaux, épices...). Les négociants européens de Venise, de Gênes et de Florence, ayant leur base commerciale à Constantinople ou Pera, y trouvaient un marché de choix pour vendre leurs draps et acheter principalement de la soie, mais également nombre d'épices. Avant la conquête, des Florentins fréquentaient déjà Brousse. En effet, en 1432-1433, Bertrandon de la Brocquière, premier écuyer tranchant du duc de Bourgogne Philippe le Bon, voyagea de Damas à Brousse en joignant une caravane de 3000 chameaux composée de marchands et de pèlerins de retour de La Mecque. Il arriva à Brousse après une cinquantaine de jours de voyage où il trouva des marchands florentins et génois de Pera qui étaient intéressés par l'achat d'épices et logea durant dix jours chez un marchand florentin ${ }^{92}$. Il s'agissait également d'un important marché de consommation de draps de laine florentins. Marco Bembo, négociant vénitien, écrivait dans une lettre de 1479 que les gens de Brousse ne connaissaient pas d'autres draps de laine que ceux de Florence, renforçant les mots de Benedetto Dei qui chantait les louanges de la supériorité de la production textile florentine ${ }^{93}$. D'après les registres de Giovanni Salviati, des marchands florentins comme Lorenzo Marsupini et Iacopo Ricardi y étaient établis et Riccardo di Francesco del Ricco s'y rendait fréquemment ${ }^{94}$. Giovanni Salviati utilisait néanmoins des agents comme Maso Fronti qui s'occupait d'y vendre des draps, ou Sandro Rucellai qui les y conduisait depuis la capitale ${ }^{95}$. Edirne, capitale des Ottomans de 1402 à 1453, était également un centre important pour le commerce des produits textiles. Des agents florentins y étaient aussi installés, tel Francesco Barducci, contact de Giovanni Salviati ${ }^{96}$, ou Giovanni Giusti, assistant d'Alessandro degli Albizzi et au service des Médicis, de Maringhi et des Michelozzi. Giovanni di Giuliano di Giovenco de' Medici, qui descendait d'une branche collatérale de la famille régnant sur Florence, y représentait l'entreprise Médicis avant 1503. En 1506, Giovanni et son frère conclurent des accords commerciaux avec Niccolò Michelozzi et Giovanni Maringhi ${ }^{97}$. Une lettre de Bonsignore Bonsignori, écrite durant son voyage au Levant en 1497-1498, livre une description de l'ancienne Andrinople, où il arriva, avec Bernardo Michelozzi, en octobre 1497. Il y indiquait la présence de boutiques et de juifs dans la ville :

A' 19 del passato per gratia di Dio a salvamento giugnemo in Andrinopoli [...] La terra d'Andrinopoli gira con le mura circha un miglio, et dentro tutta piena et è circhundata intorno da tante case che quasi in tutto si destenda quanto Firenze. Alle mura gli corrono dua fiumi, grossi ognuno più che Arno. Sonci fra dentro et fuora moltissime botteghe di panni et drappi ; et d'altri artigiani d'ogni ragione è piena. Sonci circha 50 moschee [...] Gli habitatori sono quasi tutti turchi et alchuni greci, et marrani ${ }^{98}$.

D'après ses livres comptables, et malgré quelques déplacements personnels, Giovanni Salviati utilisait plus volontiers à Edirne les services de Bernardo Giovanni ${ }^{99}$, et de manière plus sporadique, d'Alfieri Strinati, qui y était parfois présent ${ }^{100}$. Seules quatre références documentent la clientèle de Giovanni Salviati, composée d'un turc et de trois grecs.

Enfin à Gallipoli, située près du détroit des Dardanelles, deux contacts de Giovanni Salviati sont mentionnés de manière précise: Eli, un turc, qui acheta trois draps de garbo pour un montant de 3900 aspres, et Sofi Mecomedi qui fit l'acquisition de 11 draps corsivi et un 
bastardo pour 15240 aspres. Le marché de Gallipoli figure néanmoins de manière secondaire par rapport aux trois grandes places commerciales citées précédemment.

Toutefois, ce qui frappe à la lecture des registres de Giovanni Salviati est l'importance prise par les marchands juifs dans sa clientèle, désignés sous le terme giudeo ou ebreo. Ses tissus importés de Florence, composés dans une écrasante proportion de draps de laine, étaient ainsi majoritairement achetés par des négociants juifs qui possédaient des boutiques dans les principales places commerciales de l'Empire et devaient les revendre à une échelle plus locale. Ainsi, sur 19 intermédiaires dont l'origine est précisée, toutes places de commerce confondues, 13 étaient juifs. L'exemple suivant (figure 7) concerne la vente de huit draps de laine de type sopramano, datée du 26 avril 1492 à un juif du nom de Sabatino qui tenait boutique dans le Bedesten de Constantinople. Chaque drap correspondait au montant de 1550 aspres. Dans cette transaction apparaissent le nom de deux intermédiaires (sensali), également juifs, du nom de Scimito et Abraham Monbilia. Ces tissus appartenaient au marchand florentin Alfieri Strinati.

Fig. 2 - Vente de draps de laine à Sabatino, juif, le 26 avril $1492^{101}$

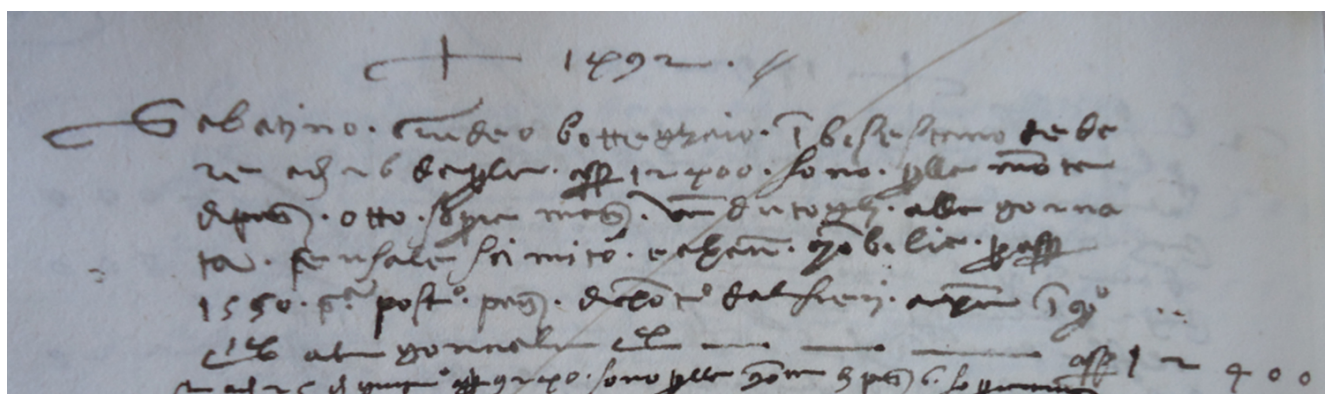

$\dagger 1492$.

Sabatino, giudeo, botteghaio i(n) Bisestano, de' dare, a dì 26 d'ap(ri)le asp. 12400 ; sono p(er) lla mo(n)ta di pan(ni) otto sop(r)aman(o) ve(n)dutogli alla g[i]ornata ; sensale Scimito e Ab(r)an Mo(n)bilia; p(er) asp. 1550 p(ezz)a ; posto pan(ni) di cho(n)to d'Alfieri av(er)e i(n) q(uest)o c.14, al g[i]ornale c.4 ----------------- asp. 12400

Les correspondances de Giovanni Maringhi présentent le même cas de figure. Les Florentins s'appuyaient sur les marchands juifs qui devinrent progressivement les intermédiaires indispensables des Européens dans le commerce intérieur de l'Empire ottoman. Après la Conquête, les sultans ottomans cherchèrent à développer un commerce local composé de Turcs, de Grecs et de juifs. Dans les livres de comptes de Giovanni Salviati figurent quelques Grecs, dont Manoli, boutiquier à Edirne, qui acheta à Giovanni Salviati vingt panni di garbo en novembre $1491^{102}$. Mais les juifs étaient nettement plus présents. Sans réelles restrictions professionnelles, en dehors de l'armée, de l'administration et des structures du pouvoir, ils occupaient une place importante au niveau commercial, notamment dans les finances et les douanes. Ils offraient des facilités aux Européens pour les tâches administratives et les diverses formalités ${ }^{103}$. Ils devinrent en toute logique les intermédiaires privilégiés entre les Latins et les Turcs, étant donné que les contacts directs étaient limités.

L'implication des marchands juifs dans le commerce en Méditerranée orientale augmenta significativement durant le $\mathrm{XVI}^{\mathrm{e}}$ siècle. Ce fut à la fois le résultat des migrations provenant d'Espagne, du Portugal et de Sicile après les expulsions promulguées à partir de $1492^{104}$, et le fruit d'une politique de protection émanant des autorités ottomanes ${ }^{105}$. Ils se spécialisèrent notamment dans le commerce en péninsule balkanique et dans ses relations avec l'Italie. De fait, divers États italiens essayèrent d'attirer les marchands juifs en leurs terres afin de stimuler leurs économies. Ce fut le cas pour Florence, en 1551, par le biais d'une invitation mentionnant explicitement les Grecs, les Turcs, les Maures, les juifs, les Arméniens et les Persans ${ }^{106}$. De plus, les conflits entre les Vénitiens et l'Empire ottoman leur permirent progressivement de substituer les Vénitiens dans leur rôle d'intermédiaires entre l'Est et l'Ouest. En effet, une fois ces guerres terminées, les Vénitiens ne regagnèrent jamais leurs positions dans le commerce avec Constantinople et les Balkans. 
Leur réussite inquiétait même les autorités vénitiennes qui démarchèrent à plusieurs reprises les pouvoirs ottomans, surtout dans les décennies 1550-1560, dénonçant la mainmise des marchands juifs sur certains approvisionnements, notamment la laine et les produits issus de l'industrie textile ${ }^{107}$. Les marchands juifs réussirent également à obtenir de nombreuses positions en tant que collecteurs des douanes dans les principales places commerciales de l'Empire : à Constantinople, Brousse, mais aussi Beyrouth ou Alexandrie. Le contrôle de positions importantes, combiné à leurs entreprises commerciales, permit aux juifs d'accroître leur position au sein de l'Empire et de progressivement détrôner la suprématie vénitienne ${ }^{108}$. Les juifs qui apparaissent dans les registres Salviati vivaient dans les villes situées sur les principaux axes commerciaux de l'Empire, près des marchés et des ports où ils tenaient leurs boutiques. Ils pouvaient également résider à Pera, mais les informations manquent à ce sujet. Avant de passer sous juridiction ottomane, Pera était considérée comme un quartier possédant un caractère occidental bien affirmé, à travers la présence des ambassades étrangères, l'implantation de marchands latins, le nombre important d'églises et l'orientation de son port vers l'Occident. Après la conquête, de nombreuses églises furent converties en mosquées et des musulmans s'implantèrent dans le quartier. Pera, au temps de Giovanni Salviati et de Giovanni Maringhi, ne possédait plus cette exclusivité chrétienne. Il s'agissait toujours du lieu de résidence de prédilections des marchands latins mais les Chrétiens d'obédience romaine, 25 ans après la Conquête, ne représentaient plus que $22 \%$ de la population de Pera. Un recensement de 1478 confirmait la présence de 535 foyers musulmans, 332 foyers catholiques, 592 foyers grecs et 32 arméniens ${ }^{109}$. Pera comptait également une population grandissante de juifs.

Giovanni Maringhi était également en collaboration avec divers juifs de Constantinople ou de Brousse, définis comme drapiers, auxquels il vendait des draps de laine ${ }^{110}$. Les sources invitent de fait à penser que les marchands florentins, tels Giovanni Salviati ou Giovanni Maringhi, étaient en contact avec la masse de boutiquiers et artisans juifs bien insérés dans l'économie urbaine des cités fréquentées, dont il est difficile d'évaluer, faute de sources complémentaires, le poids économique.

Arrivés tardivement en Méditerranée orientale, les négociants florentins surent s'insérer sur les principaux marchés de l'Empire ottoman grâce à une production textile renouvelée et orientée vers les consommateurs turcs. Ces échanges étaient motivés par l'achat de soie grège nécessaire à l'industrie de la soie florentine alors en plein essor. Quelques rares produits venaient compléter le schéma d'un commerce bilatéral très peu varié. Ce tableau vient heurter une historiographie traditionnelle faisant de la Méditerranée orientale la chasse-gardée des Génois et des Vénitiens. Les deux Républiques symbolisaient le trait d'union entre l'Orient et l'Occident par l'intermédiaire du commerce maritime. Toutefois, avec l'expansion de l'Empire ottoman et la perte de leurs possessions dans le Levant, les Génois déplacèrent leurs échanges vers l'Ouest, en particulier vers la péninsule Ibérique, et le déclin de Venise en Orient s'amorça, notamment à la suite des guerres entre la Sérénissime et l'Empire. Bien que dépourvus de flotte à l'exception d'une soixantaine d'années, Florence et ses marchands réussirent à se faire une place en Méditerranée orientale et à concurrencer directement les intérêts vénitiens.

44 Néanmoins, l'intermédiation des marchands et artisans juifs était incontournable, à la fois en raison de l'importance croissante de ce groupe sur les marchés ottomans, et de la rareté des relations directes établies par les Florentins avec les Turcs, à l'exception des hautes sphères du gouvernement. Cette situation n'était pas inconnue aux Florentins, surtout sur les marchés des pays d'Islam. Une centaine d'année auparavant, de l'autre côté de la Méditerranée, l'appui sur les juifs de Majorque ou d'Afrique du Nord, et sur les juifs convertis, représentait également le meilleur moyen de commercer avec l'Afrique septentrionale et subsaharienne, ce dont témoigne la documentation laissée par des marchands florentins établis dans l'île de Majorque et conservée dans le fonds Datini des archives de Prato ${ }^{111}$.

45 Cet article souligne ainsi à nouveau le rôle de groupes locaux de marchands dans l'économie internationale et pose les bases d'une riche étude sur le commerce interreligieux entre 
Florentins et juifs dans l'Empire ottoman. Il démontre comment les juifs, à travers leurs connexions et leurs réseaux, devinrent la clé de la pénétration commerciale des Florentins à Constantinople en devenant leurs intermédiaires privilégiés.

\section{Bibliographie}

Alberigo 1991 = G. Alberigo (dir.), Christian Unity, the Council of Ferrara-Florence 1438/1439-1989, Louvain, 1991.

Amari $1863=$ M. Amari, I diplomi arabi del R. archivio fiorentino : testo originale con la traduzione letterale e illustrazioni, Florence, 1863.

Ammannati 2008 = F. Ammannati, Note sulla decadenza dell'Arte della Lana a Firenze nel Cinquecento, dans F. Amatori et A. Colli (dir.), Imprenditorialità e sviluppo economico. Il caso italiano (secc. XIII$X X)$, Milan, 2008, p. 236-255.

Anastassiadou-Dumont 2005 = M. Anastassiadou-Dumont, Vivre ensemble en pays d'islam : territorialisation et marquage identitaire de l'espace urbain, dans Revue des mondes musulmans et de la Méditerranée, 107-110, 2005, p. 9-30. http://remmm.revues.org/2850

Anselmi 1969 = S. Anselmi, Venezia, Ragusa, Ancona tra Cinque e Seicento. Un momento della storia mercantile del Medio Adriatico, Ancône, 1969.

Apellaniz 2009 = F. Apellaniz, Pouvoir et finance en Méditerranée pré-moderne : le deuxième Etat mamelouk et le commerce ces épices (1389-1517), Barcelone, 2009.

Arbel 1995 = B. Arbel, Trading Nations. Jews and Venetians in the Early Modern Eastern Mediterranean, Leyde-New York-Cologne, 1995.

Attias 2002 = J.-C. Attias, 1453-1492. Européen et oriental $:$ le judaïsme méditerranéen à l'aube de la modernité, dans E. Benbassa et P. Gisel (dir.), L'Europe et les Juifs, Genève, 2002, p. 51-60.

Babinger 1967 = F. Babinger, Maometto il Conquistatore e il suo tempo, Turin, 1967.

Balard 1978 = M. Balard, La Romanie génoise (XII -début du XV siècle), Paris, 1978.

Baron 1952 = H. Baron, The Anti-Florentine Discourses of the Doge Tommaso Mocenigo (1414-1423): Their Date and Partial Forgery, Speculum, 27/3, 1952, p. 323-342.

Basso 1994 = E. Basso, Genova: un impero sul mare, Cagliari, 1994.

Belamarić $2013=$ J. Belamarić, Cloth and Geography: Town Planning and Architectural Aspects of the first Industry in Dubrovnik in the 15th Century, dans A. Payne (dir.), Dalmatia and the Mediterranean. Portable Archaeology and the Poetics of Influence, Leyde, 2013.

Benbassa 1992 = E. Benbassa, De l'hispanité à la turquité. Cinq cents ans d'histoire des Juifs ibériques en terre ottomane et turque, dans D. Banon (dir.), Inquisition et pérennité, Paris, 1992, p. 79-93.

Benbassa - Rodrigue 1993 = E. Benbassa et A. Rodrigue, Juifs des Balkans. Espaces judéo-ibériques, $X I V^{\mathrm{e}}-X X^{\mathrm{e}}$ siècles, Paris, 1993.

Bettarini 2007 = F. Bettarini, I Toscani al servizio della città di Ragusa (Dubrovnik) nella prima metà del Quattrocento, dans Medioevo adriatico, 1, 2007, p. 135-150.

Bisaha $2004=$ N. Bisaha, Creating East and West. Renaissance Humanists and the Ottoman Turks, Philadelphie, 2004.

Black $1985=$ R. Black, Benedetto Accolti and the Florentine Renaissance, Cambridge, 1985.

Bonazzoli 1987 = V. Bonazzoli, Ebrei italiani, portoghesi, levantini sulla piazza commerciale di Ancona intorno alla metà del Cinquecento, dans G. Cozzi (dir.), Gli ebrei e Venezia: secoli XIV-XVIII, Milan, 1987, p. 727-770.

Borromeo 2005 = E. Borromeo, Les Catholiques à Constantinople. Galata et les églises de rite latin au XVIIe siècle, dans Revue des mondes musulmans et de la Méditerranée, 107-110, 2005, p. 227-243. http://remmm.revues.org/2811

Borsook $1973=$ E. Borssok, The travels of Bernardo Michelozzi and Bonsignore Bonsignori in the Levant (1497-98), dans Journal of the Warburg and Courtauld Institutes, 36, 1973, p. 145-197.

Canaye 1986 = P. Canaye, Le voyage au Levant. De Venise à Constantinople, l'émerveillement d'un jeune humaniste (1573), M.H. Hauser (éd.), Toulouse, 1986.

Cardini 1993 = F. Cardini, Studi sulla storia e l'idea di crociata, Rome, 1993. 
Chorley 2003 = P. Chorley, Rascie and the Florentine Cloth Industry during the Sixteenth Century, dans The Journal of European Economic History, 32/3, 2003, p. 487-526.

Chorley 2004 = P. Chorley, The Volume of Cloth Production in Florence, 1500-1600: An Assessment of the Evidence, dans G. L. Fontana et G. Gayot (dir.), Wool : Products and Markets (13th to $20^{\text {th }}$ Century), Padoue, 2004, p. 551-71.

Ciccaglioni 2009 = G. Ciccaglioni, Il mare a Firenze. Interazioni tra mutamenti geografici, cambiamenti istituzionali e trasformazioni economiche nella Toscana fiorentina del '400, dans Archivio storico italiano, 167, 2009, p. 91-125.

Congourdeau $2010=$ M.-H.

Congourdeau, Pourquoi les Grecs ont rejeté l'union de Florence (1438-1439), dans B. Béthouart, M. Fourcade et C. Sorrel (dir.), Identités religieuses. Dialogues et confrontations, construction et déconstruction, Dunkerque, 2010, p. 35-46.

Coulon 2004 = D. Coulon, Barcelone et le grand commerce d'Orient au Moyen Âge. Un siècle de relations avec l'Egypte et la Syrie-Palestine (ca. 1330-ca. 1430), Madrid-Barcelone, 2004.

D'Atri 2011 = S. D'Atri, Alcuni aspetti della produzione di panni di lana a Ragusa (Dubrovnik) in età moderna, dans A. Mattone et P. Simbula (dir.), La pastorizia mediterranea: storia e diritto (secoli XIXX), Rome, 2011, p. 890-898.

Daru 1837 = P. Daru, Storia delle Repubblica di Venezia, Capolago, Tipografia Elvetica, 1837. [traduction italienne de P. Daru, Histoire de la République de Venise, Paris, 1821].

De la Broquière 1982 = B. De la Broquière, Le voyage d'outremer de Bertrandon de La Broquière, premier écuyer tranchant et conseiller de Philippe le Bon, duc de Bourgogne, Ch. Schefer (dir.), Paris, 1892 (disponible sur Gallica).

Dei 1985 = B. Dei, La cronica dall'anno 1400 all'anno 1500, R. Barducci (dir.), Florence, 1985.

Delumeau 1970 = J. Delumeau, Un ponte fra Oriente e Occidente: Ancona nel Cinquecento, Quaderni Storici, 5, 1970, p. 26-47.

Dini 1978 = B. Dini, Aspetti del commercio di esportazione dei panni di lana e dei drappi di seta fiorentini in Costantinopoli, negli anni 1522-1531, dans Studi in onore di Federigo Melis, vol. IV, Naples, 1978, p. 1-54.

Ducellier 1981 = A. Ducellier, La façade maritime de l'Albanie du Moyen Âge : Durazzo et Valona du $X I^{\mathfrak{e}}$ au $X V^{\mathfrak{e}}$ siècle, Thessalonique, 1981 (Documents et recherches sur l'économie des pays byzantins, islamiques et slaves et leurs relations commerciales au Moyen Âge, XIII).

Dursteler $2006=$ E.R. Dursteler, Venitians in Constantinople. Nation, Identity, and Coexistence in the Early Modern Mediterranean, Baltimore, 2006.

Earle $1969=$ P. Earle, The commercial development of Ancona, 1479-1551, dans The Economic History Review, 22, 1969, p. 28-44.

Efendy 1846 = Evliya Efendy, Narrative of travels in Europe, Asia and Africa in the seventeenth century (traduit du turc), R.J. von Hammer (dir.), Londres, Oriental Translation Fund of Great Britain and Ireland, 1846, 2 volumes.

Epstein 1980 = M.A. Epstein, The Ottoman Jewish Communities and Their Role in the Fifteenth and Sixteenth Centuries, Fribourg, 1980.

Fábregas García 2001 = A. Fábregas García, Estrategias de actuación de los mercaderes toscanos y genoveses en el reino nazarí de Granada a través de la correspondencia Datini, dans Serta Antiqua et Medievalia, Gênes, 2001, p. 259-304.

Faroqhi - Fleet 2013 = S.N. Faroqhi, K. Fleet (dir.), The Cambridge History of Turkey, vol. 2 The Ottoman Empire as a World Power 1453-1603, Cambridge, 2013.

Faroqhi - Veinstein 2008 = S.N. Faroqhi, G. Veinstein, Merchants in the Ottoman Empire, Leyde, 2008.

Fine Jr. 1994 = J.V.A. Fine Jr., The Late Medieval Balkans. A Critical Survey from the Late Twelfth Century to the Ottoman Conquest, Ann Arbor, 1994.

Fleet $1999=$ K. Fleet, European and Islamic Trade in the Early Ottoman State. The Merchants of Genoa and Turkey, Cambridge, 1999.

Franceschi 1993 = F. Franceschi, Oltre il « Tumulto »: I lavoratori fiorentini dell'Arte della Lana fra Tre e Quattrocento, Florence, 1993.

Galanté 1941-1942 = A. Galanté, Histoire des Juifs d'Istanbul, 2 vol., Istanbul, 1941-1942. 
Gallotta 1985 = A. Gallotta, Venise et l'Empire ottoman, de la paix du 25 janvier 1479 à la mort de Mahomet II (1481), dans Revue de l'Occident musulman et de la Méditerranée, 39, 1985, p. 113-130.

Goffman 2002 = D. Goffman, The Ottoman Empire and Early Modern Europe, Cambridge, 2002.

Goldthwaite 2003 = R.A. Goldthwaite, The Florentine Wool Industry in the Late Sixteenth Century : A Case Study, dans Journal of European Economic History, 32, 2003, p. 527-554.

Goldthwaite $2009=$ R.A Goldthwaite, The Return of a Lost Ledger to the Selfridge Collection of Medici Manuscripts at Baker Library, dans Business History Review, 83, 2009, p. 165-171.

Goldthwaite $2009^{2}=$ R.A. Goldthwaite, The Economy of Renaissance Florence, Baltimore, 2009.

González Arévalo 2012 = R. González Arévalo, Apuntes para una relación comercial velada : la República de Florencia y el Reino de Granada en la Baja Edad Media, dans Investigaciones de Historia Económica, 8, 2012, p. 83-93.

González Arévalo 2015 = R. González Arévalo, Italian Renaissance Diplomacy and Commerce with Western Mediterranean Islam: Venice, Florence, and the Nasrid Kingdom of Granada in the Fifteenth Century, dans I Tatti Studies in the Italian Renaissance, 18/1, 2015, p. 215-232.

Gulersoy 1990 = Celik Gulersoy, The Story of the Grand Bazaar, Istanbul, 1990.

Hacker 1982 = J.H. Hacker, Ottoman Policy toward the Jews and Jewish Attitudes toward the Ottomans during the fifteenth century, dans B. Braude et B. Lewis (dir.), Christians and Jews in the Ottoman Empire: the Functioning of a Plural Society. Volume I: the Central Lands, New York-Londres, 1982, p. $117-126$.

Hacker 1992 = J.H. Hacker, The Sürgün System and Jewish Society in the Ottoman Empire duringthe 15th-17th centuries, dans A. Rodrigue (dir.), Ottoman and Turkish Jewry : Community and Leadership, Bloomington, 1992, p. 1-65.

Hankins 1995 = J. Hankins, Renaissance Crusaders : Humanist Crusade Literature in the Age of Mehmed II, dans Dumbarton Oaks Papers, 49, 1995, p. 111-207.

Heyd 1885-1886 = W. Heyd, Histoire du commerce du Levant au Moyen Âge, Leipzig, 1885-1886.

Hoshino $1980=\mathrm{H}$. Hoshino, L'arte della lana in Firenze nel Basso Medioevo: il commercio della lana e il mercato dei panni fiorentini nei secoli XIII-XV, Florence, 1980.

Hoshino $2001=\mathrm{H}$. Hoshino, Industria tessile e commercio internazionale nella Firenze del tardo Medioevo, F. Franceschi, et S. Tognetti (éd.), Florence, 2001.

Hoshino - Mazzaoui 1985-1986 = H. Hoshino, M. Mazzaoui, Ottoman Markets for Florentine Woolen Cloth in Late Fifteenth Century, dans International Journal of Turkish Studies, 3, 1985-1986, p. 17-31.

Houssaye Michienzi 2013 = I. Houssaye Michienzi, Datini, Majorque et le Maghreb $\left(14^{\mathrm{e}}-15^{\mathrm{e}}\right.$ siècles $)$. Réseaux, espaces méditerranéens et stratégies marchandes, Leyde, 2013.

Inalcik 1973 = H. Inalcik, The Ottoman Empire. The Classical Age, 1300-1600, Londres, 1973.

Inalcik $1980=\mathrm{H}$. Inalcik, The Hub of the City : the Bedestan of Istanbul, dans International Journal of Turkish Studies, 1, 1980, p. 1-17.

Inalcik $1989=\mathrm{H}$. Inalcik, Jews in the Ottoman Economy 1450-1500, dans C.E. Bosworth et C. Issawi (dir.), Essays in Honor of Bernard Lewis. The Islamic World, Princeton, 1989, p. 513-555.

Inalcik 1991 = H. Inalcik, Ottoman Galata, 1453-1553, dans E. Eldem (dir.), Première rencontre internationale sur l'Empire ottoman et la Turquie moderne, Istanbul-Paris, 1991, p. 17-105.

Inalcik - Quataert $1994=\mathrm{H}$. Inalcik et D. Quataert, An Economic and Social History of the Ottoman Empire, vol.1 : 1300-1600, Cambridge, 1994.

Janin 1946 = R. Janin, Les sanctuaires des colonies latines à Constantinople, dans Revue des études byzantines, 4, 1946, p. 163-177.

Judde de Larivière 2008 = C. Judde de Larivière, Naviguer, commercer, gouverner. Économie maritime et pouvoirs à Venise (XVe-XVIe siècles), Leyde, 2008.

Krekić 1980 = B. Krekić, Dubrovnik, Italy and the Balkans in the late Middle Ages, Londres, 1980.

Lewis 1963 = B. Lewis, Istanbul and the Civilization of the Ottoman Empire, Princeton, 1963.

Levy 2002 = A. Levy (dir.), Jews, Turks, Ottomans: A Shared History, Fifteenth Through the Twentieth Century, Syracuse (NY), 2002.

Lewis 1989 = B. Lewis, Juifs en terre d'Islam, Paris, 1989. 
Mallet $2013=\mathrm{L}$. Mallet, Quand le présent fait le passé : modalité et finalité du récit de l'harmonie turcojuive, dans Cahiers de la Méditerranée, 86, 2013, p. 301-317. http://cdlm.revues.org/6882

Mallett 1967 = M.M. Mallett, The Florentine galleys in the fifteenth century, Oxford, 1967.

Mansel 1997 = P. Mansel, Constantinople. La ville que désirait le monde 1453-1924, Paris, 1997.

Mantran 1994 = R. Mantran, Istanbul au siècle de Soliman le Magnifique, Paris, 1994.

Mantran 1996 = R. Mantran, Histoire d'Istanbul, Paris, 1996.

Masi 1941 = G. Masi, Statuti delle colonie fiorentine all'estero (secoli XV-XVI), Milan, 1941.

Meli 2009 = P. Meli, Firenze di fronte al mondo islamico. Documenti su due ambasciate (1487-1489), dans Annali di Storia di Firenze, 4, 2009, p. 241-271.

Milano 1963 = A. Milano, Storia degli ebrai in Italia, Turin, 1963.

Mitler 1979 = L. Mitler, The Genoese in Galata: 1453-1682, dans International Journal of Middle East Studies, 10, 1979, p. 71-91.

Müller 1879 = G. Müller, Documenti sulle relazioni delle città toscane coll'Oriente cristiano e coi Turchi, fino all'anno 1531, Florence, 1879.

Munro 2007 = J.H. Munro, I panni di lana, dans F. Franceschi, R.A. Goldthwaite et R.C. Mueller (dir.), Il Rinascimento italiano e l'Europa, IV, Commercio e cultura mercantile, Trévise-Costabissara, 2007, p. $105-141$.

Pagnini del Ventura 1765-1766 = F. Pagnini del Ventura, Della decima e di varie altre gravezze imposte dal comune di Firenze, della moneta e della mercatura de' Fiorentini fino al secolo XVI, LisbonneLucques, 1765-1766.

Pegolotti 1936 = F.B. Pegolotti, La Pratica della Mercatura, A. Evans (éd.), Cambridge (Mass.), 1936.

Pistarino 1984 = G. Pistarino, La caduta di Costantinopoli : da Pera genovese a Galata turca, dans La storia dei Genovesi, 5, 1984, p. 7-47.

Ravid 1991 = B. Ravid, A Tale of Three Cities and their Raison d'Etat: Ancona, Venice, Livorno, and the Competition for Jewish Merchants in the Sixteenth Century, dans Mediterran Historical Review, 6, 1991, p. 138-162.

Ricci 2008 = G. Ricci, I Turchi alle porte, Bologne, 2008.

Rozen 2002 = M. Rozen, History of the Jewish Community in Istanbul: The Formative Years, 1453-1566, Leyde, 2002.

Schwoebel $1965=$ R. Schwoebel, Coexistence, Conversion and the Crusade Against the Turks, dans Studies in the Renaissance, 12, 1965, p. 164-187.

Schwoebel 1967 = The Shadow of the Crescent: the Renaissance image of the Turk 1453-1517, Nieuwkoop, 1967.

Setton 1978 = K.M. Setton, The Papacy and the Levant (1204-1571), vol II. The Fifteenth Century, Philadelphie, 1978.

Shaw 1991 = S. Shaw, The Jews of the Ottoman Empire and the Turkish Republic, New York, 1991.

Shmuelevitz 1984 = A. Shmuelevitz, The Jews of the Ottoman Empire in the Late Fifteenth and Sixteenth Centuries : Administrative, Economic, Legal and Social Relations as Reflected in the Responsa, Leyde, 1984.

Sopracasa $2011=$ A. Sopracasa, Les marchands vénitiens à Constantinople d'après une tariffa inédite de 1482, dans Studi veneziani, 63, 2011, p. 49-220.

Simon 1984 = B. Simon, Contribution à l'étude du commerce vénitien dans l'empire ottoman au milieu du seizième siècle (1558-1560), dans Mélanges de l'École française de Rome. Moyen Âge-Temps modernes, 96-2, 1984, p. 973-1020.

Stefini $2010=$ T. Stefini, Irregolarità e rapporti di forza nella Dalmazia del Cinquecento, dans Studi veneziani, 59, 2010, p. 625-652.

Tanzini 2010 = L. Tanzini, Il Magnifico e il Turco. Elementi politici, economici e culturali nelle relazioni tra Firenze e Impero Ottomano al tempo di Lorenzo de' Medici, dans Rivista dell'Istituto di Storia dell'Europa Mediterranea, 4, 2010, p. 271-289.

Tenenti 1985 = A. et B. Tenenti, Il prezzo del rischio. L'assicurazione mediterranea vista da Ragusa, 1563-1591, Rome, 1985.

Theunissen 1998 = H.P.A. Theunissen, Ottoman-Venetian Diplomatics : The 'Ahd-names. The Historical Background and the Development of a Category of Political Commercial Instruments together with an 
Annotated Edition of a Corpus of Relevant Documents, dans Electronic Journal of Oriental Studies, 1/2, 1998, p. 1-698.

Tognetti 2003 = S. Tognetti, Da Figline a Firenze. Ascesa economica e politica della famiglia Serristori (secoli XIV-XVI), Florence, 2003.

Tognetti 2009 = S. Tognetti, Il banco Cambini. Affari e mercati di una compagnia mercantile-bancaria nella Firenze del XV secolo, Florence, 1999.

Tuson 1856 = E.W.A. Tuson, The British consul's manual : being a practical guide for consuls, as well as for the merchant, shipowner, and master mariner, in all their consular transactions, Londres, 1856.

Vatin 1987 = N. Vatin, Itinéraires d'agents de la Porte en Italie (1483-1495). Réflexions sur l'organisation des missions ottomanes et sur la transcription turque des noms de lieux italiens, Turcica, 19, 1987, p. 29-50.

Veinstein 1992 = Gilles Veinstein, L'Empire ottoman depuis 1492 jusqu'à la fin du XIX siècle, dans H. Méchoulan (dir.), Les Juifs d'Espagne, histoire d'une diaspora, 1492-1992, Paris, 1992, p. 367-381.

Yerasimos 1991 = S. Yerasimos, Les voyageurs dans l'Empire ottoman (XIV $-X V I^{\mathrm{e}}$ siècles), Ankara, 1991.

Yerasimos $1995=\mathrm{S}$. Yerasimos, La communauté juive d'Istanbul à la fin du XVI siècle, dans Turcica, 27, 1995, p. 101-130.

\section{Notes}

1 La seule étude de vaste ampleur et bien documentée concerne les activités des Florentins au Maghreb : voir Houssaye Michienzi 2013. Voir également les articles de Raúl González Arévalo et Adela Fábregas García pour le sultanat nasride de Grenade, et de Francisco Apellaniz pour le sultanat mamelouk. Cf. Apellaniz 2009, Fábregas García 2001, González Arévalo 2012 et 2015.

2 Voir Coulon 2004.

3 La conquête de Pise par Florence en 1406 n'apporta pas de véritables changements. Ce n'est qu'en 1421, à travers l'achat de Porto Pisano et de Livourne, que les Florentins se rendirent indépendants des marines étrangères et mirent sur pied leur propre flotte. La fonction des Consoli del Mare fut créée, dont les attributions comprenaient la création d'un arsenal maritime, la construction et l'équipement de galées de commerce ainsi que la surveillance des ports. Différents décrets parus les années suivantes, en 1422 et en 1423, leur conférèrent des pouvoirs dans le domaine diplomatique notamment à travers l'envoi d'ambassades et la nomination de consuls chargés de veiller aux intérêts florentins. Les premières délégations furent envoyées à Tunis, à Alexandrie et à Constantinople dans le but à la fois d'ouvrir des négociations et de mettre en place les nouveaux services de navigation.

4 Voir Mallett 1967, Ciccaglioni 2009.

5 Voir Alberigo 1991. Et plus récemment Congourdeau 2010.

6 Müller 1879, Janin 1946.

7 Cf. Müller 1879, p. 169-172 : In logia dominorum Catalanorum [...] ego Petrus Samoyragensis publicus notarius et curie consulatus dominorum Catalanorum et Florentenorum in regia urbe Constantinopolis cancellarius.

8 Salviati, I, 222, Florence, Libro di ricordanze L, fol. $64 \mathrm{v}^{\circ}$.

9 Lorenzo Tanzini, avec un questionnement historiographique renouvelé et à la lumière de publications récentes de lettres de Lorenzo de Médicis et des ambassadeurs florentins à la cour de Naples, reprend le thème déjà développé dans les travaux de Frank Babinger au sujet de la politique pro-turque du Magnifique à la fin du règne de Mehmed II et au début de celui de son fils Bajazet II. Voir Tanzini 2010, Babinger 1967. Voir également Ricci 2008. Des échanges épistolaires témoignent des bonnes relations entre Lorenzo de Médicis et le sultan Mehmed II, notamment après l'arrestation de Bernardo Bandini, l'assassin de Giuliano de Médicis (frère de Lorenzo) lors de la conjuration des Pazzi de 1478, qui s'était réfugié à Constantinople.

10 Voir Bisaha 2004, Black 1985, Cardini 1993, Hankins 1995. Voir également les travaux de R. Schwoebel 1965 et 1967. 
11 Voir Black 1985.

12 Voir Gallotta 1985.

13 D'après les dires de Benedetto Dei, à considérer néanmoins avec précaution, certains Florentins auraient également cherché à accentuer l'animosité du sultan envers les Vénitiens en interceptant des lettres écrites par des marchands vénitiens afin de les mettre sous les yeux du sultan, tout en donnant parallèlement au sultan toutes les informations lui permettant de nuire aux Vénitiens. En récompense, le sultan emprisonna certains sujets vénitiens dont les maisons échurent ainsi à leurs rivaux florentins. Cf. Dei 1985, p. 160 (année 1461) : «' 'l gran turcho $[\ldots]$ pe ll'amore ched e' portava a' Fiorentini, fe' pigliare tutti I Viniziani e mettergli in ferri ; e dapoi gli fe' chagiare delle chase e die' lle a' Fiorentini ».

14 Goffman 2002, p. 176 : «During the first years of the war, business between Ottomans and Florentines replaced the lost Veneto-Ottoman nexus, and flourished».

15 Voir Müller 1879.

16 Le manuscrit, de lecture difficile, est conservé dans le vaste fonds Selfridge de la Baker Library à Harvard. Je remercie la Baker Library et la Villa I Tatti (Florence) d'avoir mis le microfilm à ma disposition. Voir à ce sujet Goldthwaite 2009.

17 Voir Dei 1985.

18 Voir Hoshino 2001, en particulier : Il commercio fiorentino nell'Impero ottomano: costi e profitti negli anni 1484-1488, et Alcuni aspetti del commercio dei panni fiorentini nell'Impero ottomano ai primi del '500. L'auteur se focalise sur les draps de laine florentins qui intéressaient le marché ottoman. Il ne fait cependant aucune remarque concernant la présence et les contacts de ces marchands dans l'Empire.

19 Voir Dini 1978.

20 En plus des sources formant la base du corpus utilisé et détaillées plus en avant, il a été fait usage de sources imprimées relatives aux accords commerciaux et aux relations de voyages, notamment : Amari 1863, De la Broquière 1982, Canaye 1986, Müller 1879.

21 Fonds d'archives comprenant des milliers de registres et de documents des XIV ${ }^{\text {e }}$ $\mathrm{XVII}^{\mathrm{e}}$ siècle, dont plusieurs dizaines de séries comptables complètes, déposé à la Scuola Normale Superiore de Pise depuis 1982. Cet immense fonds est actuellement mis en valeur par une équipe de chercheurs dans le cadre du projet ANR ENPrESa (http:// salviati.hypotheses.org).

22 Ou Galata, un quartier situé au nord de la ville de Constantinople, de l'autre côté de la Corne d'Or, maintenant connu sous le nom de Beyoğlu. Il s'agissait à l'origine d'une « colonie » génoise. Quelques jours après la conquête turque, les Génois remirent les clefs de leur ville au sultan qui intégra pacifiquement Pera dans la nouvelle capitale ottomane en tant qu'un de ses quatre districts (avec Stamboul -la vieille ville-, Eyyub et Ûsküdar ou Scutari. Voir Borromeo 2005, Inalcik 1991, Mitler 1979, pistarino 1984.

23 Scuola Normale Superiore di Pisa, Archivio Salviati (désormais Salviati), série I, registre 397, Constantinople, fol. XXIX, lettre écrite le 16 novembre 1493 à Pera : « Noi sottoscritti $\mathrm{m}(\mathrm{er})$ chatanti faciamo fede chome trovandosi la buona memoria di Giovan(ni) di Marcho Salviati amalato di pesta in letto ». La lettre contient les noms de marchands florentins établis alors dans l'Empire ottoman.

24 Salviati, I, 397, Constantinople, Debitori e Creditori, fol. 55d. : « Di qui avanti si riddurano t(ut)te le schriptt(ure) di Giovanni Salviati, a chuy Idio p(er)doni p(er) non es(ser)e chontinovati i sua lib(r)i e schriptt(ure), li qualy avem(m)o d'Alfiery Strinati, (un)o degli seghutori del testam(en)to, de li quali sia nota qui ap(r)exo p(er) me, Ant(oni)o Miniati, churatore de la 'redità giacente di deto Giovan(n)i, qui, in Fi(ren)ze ».

25 Salviati, I, 398, Constantinople, Giornale, fol.9v., 07/07/1492 : «Fa debitore Alfieri e creditore chassa di d.351 d'oro [...] vagliono asp. 52 p(er) d. ».

26 Salviati, I, 398, Constantinople, Giornale, fol.11v., 19/07/1492.

27 Du nom du couvent de San Martino, au cœur de la ville (entre le Duomo et le Palazzo Vecchio) où la plupart des ateliers étaient installés.

28 Le nom tient son origine d'une région d'Afrique du Nord, le Gharb, d'où provenait une laine qui servait à la confection de ce type de tissus. 
29 Au sujet de la production florentine de draps de laine, se référer à Chorley 2003 et 2004, Goldthwaite 2003, Hoshino 2001 (notamment p. 125-135), Munro 2007.

30 Pegolotti 1936, notamment p. 38.

31 Voir Daru 1837, t. III, Libro XIII, p. 128.

32 Voir Baron 1952.

33 Voir Dei 1985, p. 94-100.

34 Voir Hoshino 1980 et 2001, Hoshino - Mazzaoui 1985-1986, Goldthwaite 2009²

35 Goldthwaite $2009^{2}$, p. 186

36 Voir Hoshino 1980, p. 235 et suivantes. Au sujet de la chute de la production et des difficultés qui marquèrent le début du XVe siècle voir également Ammannati 2008, Chorley 2003, F Franceschi 1993, Munro 2007.

37 Salviati, I, 263, Florence, Libro grande debitori e creditori, 1486-1500.

38 Salviati, I, 399, Constantinople, fol. 100r. à fol. 101r. Il s'agit de la « copia dell'dagio de' panni CLXV di più sortte avuti da' Salviati di Firenze in balle XL come di sotto si dice ». L'intitulé indique une quantité de 165 draps de laine qui sont en fait 160 draps à vendre et cinq draps pour l'emballage.

39 Dans les ricordanze de certains de ces registres se trouvent des rubriques concernant les ventes de draps présentées en deux étapes : les ventes de draps, et les ventes des draps qu'ils ont produits. Ceci indique que l'atelier, en plus d'effectuer sa propre production, se livrait à l'achat de draps en vue de leur commercialisation.

40 Hoshino 1980, p. 243.

41 Voir Mallett 1967.

42 Florence et les Consuls de la mer délibérèrent que le voyage du Levant se fasse désormais avec deux galées au lieu d'une. Cf. Müller 1879, p. 293 : « considerato che la ghalea, la quale debbe ire alle parti di Levante, va male sola, per rispecto delle poche sichurtà sono in questi mari $[\ldots]$ si provede, che per lo viaggio di dee fare con una ghalea solamente, si faccia con due».

43 Inalcik - Quataert 1994, p. 231-233.

44 Voir Borsook 1973.

45 Müller 1879, p. 216.

46 Valona, ou Avlonva située sur la rive albanaise du canal d'Otrante, était sous domination ottomane depuis 14@t chef-lieu de sandjak, division administrative, judiciaire et militaire de l'Empire ottoman. Il s'agissait alors du seul port important dont les Ottomans disposaient sur l'Adriatique. Voir Fine Jr. 1994 (chapitre 10), Vatin 1987. Pour la période antérieure à la domination turque, consulter Ducellier 1981.

47 Salviati, I, 399, Constantinople, fol. 98d. : « Copia d'uno chonto dateci dateci Dimittri $\mathrm{n}$ (ost)ro gharzone in Pera di spese fatt(e) a balle XLII di panni sam(ar)ttini e gharbi, e chasse IIII $^{\circ}$ di drappi chon oro e sa(n)za oro di ragione di [...] Alaman(n)o e Iac(op)o Salviati e c(ompagnia) di Fire(n)ze e P(r)iore Strinati e a Strinato Strinati, da Lleccio insino in Pera, e p(r)ima chondotti p(er) Dimittri n(ost)ro gharzone, e lui à fatto le sottoscritte spese e a cciaschuno de' sudetti se n'è dato la p(ar)t(i)t(a)».

48 Ibid.: « p(er) nolo da San Chataldo alla Valona duc. XI d(enar)o in o(ro) viniziani v(alutat)o asp. $51 \mathrm{p}(\mathrm{er})$ duc(ato) $\gg$.

49 Ibid. : « $\mathrm{P}(\mathrm{er})$ aghugl[i]o dalla Velona in Chastoria di chavalli XXIIII ${ }^{\circ} »$.

50 Ibid. : «P(er) aghugl[i]o di Chastoria in A(n)drinop(o)li di chavelli [sic] XXIIII ${ }^{\circ}$, asp. 115, soma in t(ut)to asp. $2760 »$.

51 Ibid. : « P(er) aghugl[i]o d'Andrinop(o)li in Pera e portta di Ghonstanop(o)li in t(ut)to asp. $1400 »$.

52 Ce registre est également conservé dans le fonds Salviati (série I, 70). Il fut à la base d'un article de Bruno Dini, 1978.

53 En mars 1492. Salviati, I, 398, Constantinople, Giornale, fol. 3r. et I, 397, Constantinople, Debitori e Creditori, fol. 9.

54 Simone di Bartolomeo Zati était également présent à partir de 1503 à Pera où il travaillait pour le compte des Serristori. Voir Tognetti 2003, p. 163. 
55 En août 1492. Salviati, I, 398, Constantinople, Giornale, fol. 16r. et I, 397, Constantinople, Debitori e Creditori, fol. 9.

56 En février 1493. Salviati, I, 398, Constantinople, Giornale, fol. 27r. et I, 397, Constantinople, Debitori e Creditori, fol. 40.

57 Voir à ce sujet les travaux de Krekić 1980. Voir également Anselmi 1969, Bonazzoli 1987, D’Atri 2011, Delumeau 1970, Earle 1969. Pour les relations entre Florence et Raguse au début du XV' siècle, voir Bettarini 2007.

58 Au sujet de Raguse et des côtes dalmates, voir notamment Stefini 2010.

59 Belamarić 2013, D'Atri 2011.

60 Salviati, I, 262, Florence, Ricordanze .BB. 1486-1491 et I, 263, Florence, Libro grande Debitori e Creditori .BB.,1486-1500.

61 Salviati, I, 397, Constantinople, Debitori e Creditori, fol. 22.

62 Voir Tenenti 1985.

63 Voir Yerasimos 1991. C'était également l'itinéraire choisi par les agents de la Porte se rendant à Florence, à Rome ou dans l'Europe du Nord-Ouest. Cf. Vatin 1987.

64 Au sujet des Génois et des Vénitiens à Constantinople, voir notamment Balard 1978, Basso 1994, Dursteler 2006, Faroqhi - Fleet 2013, Faroqhi - Veinstein 2008, Judde de Larivière 2008, Fleet 1999, Sopracasa 2011, Theunissen 1998.

65 Goldthwaite 2009, p. 184. Nous ne trouvons pas de trace de ce consul dans les documents transcrits par Giuseppe Müller. Seul Benedetto Dei y fait référence.

66 Voir Tognetti 2009, p. 235-237. Le banco Cambini disposait également d'un autre correspondant à Constantinople en 1461-1462: Bartolomeo di Domenico Giugni (p. 228).

67 Voir Meli 2009.

68 Voir également à ce sujet Pagnini del Ventura 1765-1766, vol. 2, partie 3 « sul commercio dei Fiorentini », section 3, chapitres 1 à 6 .

69 Voir Heyd 1885-1886, t.2, p. 344.

70 Le premier adh-name (capitulations) fut octroyé aux Florentins par Mehmed II peu après la conquête, même si aucune trace de cet accord n'est conservée dans les archives. Néanmoins, sans l'existence de capitulations, les Florentins n'auraient pu rester à Constantinople. Voir Inalcik 1991, p. 62 : «The first formal ahd-name was granted to the Florentines by Mehmed II, the text of which has not been discovered. Without such an instrument the Florentine colony could not have remained in Galata ». Néanmoins, dans un document daté du 3 décembre 1455, transcrit par Giuseppe Müller, la République de Florence remercie le sultan Mehmed II de ses bonnes dispositions à l'égard des Florentins, le priant de bien vouloir concéder à ses ressortissants un libre accès dans les terres placées sous son autorité. De même, le 8 juin 1469, Florence remercie le sultan Mehmed II des faveurs accordées aux marchands florentins dans son royaume. Cf. Müller 1879, p. 182 et 211.

71 Inalcik 1991, p. 65 : «This document [1427] is decisive proof that Bayezid II and Selim I granted ahd-names to the Florentine Signora guaranteeing the establishment of a Florentine community in Galata under a Baylos on the same basis as the Venetians ». Nous nous référons aux articles des capitulations traduits dans ce même article p. 63-65.

72 Baker Library Harvard, Selfridge collection, MS 547, fol. 64, 1501.

73 Le sandjak-bey (sangiacco) était à la tête du sandjak (sangiaccato). Il recevait ses instructions directement du sultan.

74 Voir Müller 1879, p. 242, 253, 342-343 et Masi 1941, p. 154.

75 Ce tableau, non exhaustif, présente différents consuls et ambassadeurs envoyés par la République florentine à Constantinople au $\mathrm{XV}^{\mathrm{e}}$ siècle et au début du $\mathrm{XVI}^{\mathrm{e}}$. Il a été conçu au fil de lectures. Voir Setton 1978, p. 521, en plus des ouvrages déjà cités en bibliographie.

76 Bernardo Bandini, assassin de Giuliano de' Medici (frère de Lorenzo), lors de la conjuration des Pazzi, le 26 avril 1478, s'était réfugié à Constantinople. Il y fut arrêté en avril/mai 1479 et incarcéré. Extradé à Florence, il fut pendu dans le palais du Bargello le 29 décembre 1479.

$77 \mathrm{Il}$ aurait auparavant été consul des Anglais à Pise, en 1485. Voir Tuson 1856, p. 3.

78 Certainement le même Carlo Baroncelli qui fut un ancien consul de la nation florentine à Constantinople, élu en 1472. 
79 Salviati, I, 398, Constantinople, Giornale, fol. 24v. : « Fa debitore Lorenzo Ridolfi di asp. 180 per pig[i]one di mesi sei del maghazino di sepugli »; Salviati, I, 397, Constantinople, Debitori e Creditori, fol. 28g. : il est débiteur de 30 aspres « $\mathrm{p}(\mathrm{er}) \mathrm{pig}[\mathrm{i}] \mathrm{one}$ di $\mathrm{I}^{\circ}$ mese del maghazino di saponi ».

80 Voir la mention d'une opération datée du 19 juillet 1492 concernant 131 sacs de savons dans Salviati, I, 398, Constantinople, Giornale, fol. 11v.

81 Salviati, I, 398, Constantinople, Giornale, fol. 5r. : « Fa debitore chassa e creditore Ricardo asp. 13863 lasciaromi i(n) $I^{\circ}$ sachetto sugelato qua(n)do andò i(n) Andrinop(o)li ». Cette entrée date du 4 mai 1492. Edirne est située à près de 220 kilomètres de Constantinople.

82 Salviati, I, 398, Constantinople, Giornale, fol. 14v. : « q(ua)ndo andò i(n) B(ur)sia ». Cette entrée date du 11 août 1492. Brousse est située à environ 90 kilomètres de Constantinople, mais à près de 240 kilomètres à travers l'utilisation de voies terrestres.

83 Salviati, I, 397, Constantinople, Debitori e Creditori, fol. 11g.

84 Ibid., fol. $17 \mathrm{~d}$.

85 Ibid., fol. 27s. : « a L(eonard)o Salucci c(onsol)o p(er) pig[i]one di I ${ }^{a}$ casetta p(er) $I^{\circ}$ an(n)o a rag[i]one di aspri 20 al mese ». Il devait s'agir de Leonardo Salvucci, ancien consul élu en 1481 mais qui n'était alors plus en exercice.

86 Ibid., fol. $27 \mathrm{~s}$. : « p(er) pig[i]one di I $\mathrm{I}^{\circ}$ an(n)o dello ortto de' frati ».

87 Voir par exemple Salviati, I, 397, Constantinople, Debitori e Creditori, fol. 27g. et 35g.

88 Voir Inalcik 1980 et Gulersoy 1990. Dans un style imagé, philip Mansel offre une description du Bazar de Constantinople et de ses abords, grouillant de marchands de diverses origines : Mansel 1997, p. 142-143.

89 Voir Anastassiadou-Dumont 2005.

90 Voir notamment Inalcik 1973, Lewis 1963, Mantran 1996.

91 Salviati, I, 397, Constantinople, Debitori e Creditori, fol. 48g.

92 Voir De la Broquière 1982, p. 131-137. Une description plus tardive de Brousse figure dans la relation des voyages d'Evliya Efendy (Efendy 1846).

93 Voir Sopracasa 2011, p. 111 et Pagnini 1765-1766, p. 241.

94 Voir leurs comptes figurant dans Salviati, I, 397, Constantinople, Debitori e Creditori, fol. 11,12 et 16 .

95 Salviati, I, 397, Constantinople, Debitori e Creditori, fol. 36d. : « Pan(ni) di cho(n)to fi Franc(esc)o Gherardi proprio, debono av(er)e [...] asp. 4800 ; sono p(er) lla mo(n)ta di 4 cholonbin(i) chorsivi ve(n)dé i(n) B(ur)sia Maso Fro(n)ti p(er) me più dì sono [...] E detti pan(ni) cho(n)dusse Sa(n)dro Rucellai ».

96 Voir ses comptes figurant dans Salviati, I, 397, Constantinople, Debitori e Creditori, fol. 11 et 59 .

97 Borsook 1973, p. 157-158.

98 Ibid., p. 183-184.

99 Salviati, I, 397, Constantinople, Debitori e Creditori, fol.11g. : « Franc(esc)o B(ar)ducci de' dare [...] asp. 629 auti p(er) me i(n)n A(n)drinopoli da B(er)nardo G[i]ovan(ni) ». Un compte détaillant les frais contractés par la vente de tissus à Edirne permet de confirmer sa fonction en tant qu'agent en ce lieu ; il s'acquitta en effet du montant des taxes et des frais de douane pour le transport et la vente de 48 draps de laine (Salviati, I, 397, Constantinople, Debitori e Creditori, fol.6g.)

100 Giovanni Salviati le rencontra à Edirne pour solder une dette. Voir Salviati, I, 397, Constantinople, Debitori e Creditori, fol.32d. : " Alfieri di cho(n)t(r)o, de' av(er)e [...] asp. 1000 auti da lui in cho(n)ta(n)ti, qua(n)do a(n)dò in A(n)drinop[o]li ».

101 Salviati, I, 397, Constantinople, Debitori e Creditori, fol.14g.

102 Salviati, I, 398, Constantinople, Giornale, fol.1v. : «Fa debitore Manoli, grecho, e creditore pan(n)i di Franc(esc)o Gherardi di asp. 24300 ; sono p(er) lla monta di pan(n)i 20 di g(arb)o bag(nia)ti e cinati ; te(m)po mesi 3, paghati i(n) cinq(u)e, c[i]oè 12 fra tt(urchin)i e sbiadati, 4 paonazi, 4 festichin(n)i ». Il était boutiquier à Edirne (Salviati, I, 397, Constantinople, Debitori e creditori, fol.2g : «Manoli, grecho, botteghaio i(n) Andrinop[o]li ». 
103 Voir Mantran 1994, p. 58 : « les Juifs, qui se révèlent de plus en plus comme intermédiaires entre Francs et Ottomans, se font une spécialité de faciliter aux Occidentaux les démarches indispensables, les formalités nécessaires pour l'obtention des permis de navigation, de déchargement ou de chargement des marchandises, etc. ».

104 Que ce soit à partir de sources ottomanes ou bien de chroniques juives, les historiens contemporains estiment qu'entre 10 et 12000 chefs de famille juifs espagnols rejoignirent l'Empire ottoman au cours du XVI siècle. Soit, si on retient un facteur 5, ce mouvement migratoire sur plusieurs générations aurait concerné 50 à 60000 personnes. Voir Inalcik 1989, p. 515. En référence à Don Isaac Abravanel qui évoque le nombre de familles juives d'Espagne passées par l'Italie avant de rejoindre l'Empire, voir Veinstein 1992, p. 365 et Yerasimos 1995, p. 104 et 106, où l'auteur arrive à une évaluation d'environ 3,3 personnes par foyer fiscal.

105 Voir Arbel 1995, en particulier le premier chapitre.

106 Voir Milano 1963, p. 267-268 et Ravid 1991, p. 144.

107 Voir Simon 1984.

108 Sur les juifs dans l'Empire ottoman, voir : Attias 2002, Benbassa 1992, Benbassa Rodrigue 1993, Epstein 1980, Galanté 1941-1942, Hacker 1982 et 1992, Levy 2002 (première partie), Lewis 1989, Mallet 2013, Rozen 2002, Shaw 1991, Shmuelevitz 1984.

109 Inalcik 1991, p. 97.

110 Baker Library Harvard, Selfridge collection, MS 547, fol. 68, 1501, quelques exemples :

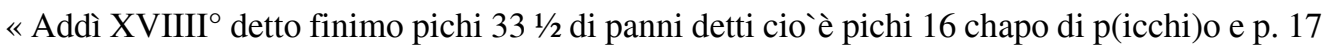
$1 / 2$ di panno rosetto pure bastarddi a Bigliamino Allamano eb(r)eo drappieri in Chostantinopoli p(er) asp. 1302, t(ut)to p(er) tenpi usati [...] Addì 25 d(ett)o finimo panni IIII ${ }^{\circ}$ sop(r)a a Iusuffio di Bacciaci, eb(r)eo drappieri in Chostantinopoli p(er) asp. 1587 p(ezz)a, tenpi ordinari, sensale Aionne Lungho. E addì detto finimo a detto Iusuffio panni III bastardi p(er) asp. 1400 p(ezz)a, tenppo ordinari, e più p. 19 1/2 di panno rosetto di detti chonti. Di B(ur)sia abiamo e finito di poi pann(i) $\mathrm{XI}^{\circ} \operatorname{sop}(\mathrm{r}) \mathrm{a}$ a Isache Ttapiero, eb(r)eo drappieri di lì p(er) asp. 1595 p(ezz)a ».

111 Voir Houssaye Michienzi 2013.

\section{Pour citer cet article}

Référence électronique

Ingrid Houssaye Michienzi, «Les milieux d'affaires florentins, le commerce des draps et les marchés ottomans à la fin du $\mathrm{XV}^{\mathrm{e}}$ et au début du $\mathrm{XVI}^{\mathrm{e}}$ siècle », Mélanges de l'École française de Rome - Moyen Âge [En ligne], 127-2 | 2015, mis en ligne le 14 décembre 2015, consulté le 14 décembre 2015. URL : http://mefrm.revues.org/2753

\section{À propos de l'auteur}

Ingrid Houssaye Michienzi

The Harvard University Center for European Renaissance Studies - Villa I Tatti, Florence, ingridhoussaye@yahoo.fr

\section{Droits d'auteur}

(C) École française de Rome

\section{Résumés}

Les activités des négociants florentins, grâce à d'importants dépôts d'archives sur lesquels se sont penchées plusieurs générations d'historiens, sont relativement bien connues, mais il est des lacunes historiographiques persistantes qui nécessitent d'être comblées. Ainsi, si les relations commerciales de Venise, de Gênes, d'Ancône ou de Raguse avec l'Empire ottoman ont fait l'objet de nombreux écrits, Florence et ses marchands sont quasiment 
absents de l'historiographie. Ce présent article a ainsi pour but à la fois de combler ce vide historiographique et de bouleverser l'image classique d'une Méditerranée orientale dominée par Gênes et Venise et leurs constantes rivalités. Le corpus réuni, composé de documents issus de la pratique des affaires - registres de comptes et correspondances - offre d'abondantes informations sur le commerce qui était alors effectué dans l'Empire par les marchands florentins et permet d'analyser leurs mécanismes d'insertion sur les plus importants marchés de l'Empire ottoman à la fin du XV siècle et au début du XVI'.

Thanks to relevant archives, which have been studied by several generations of historians, the activities of the Florentine traders are relatively well known, but there are persistent historiographical gaps, which require to be filled. If the business connections of Venice, Genoa, Ancona or Ragusa with the Ottoman Empire were the object of numerous studies, Florence and its traders are almost absent of the historiography. This present article aims at filling this historiographical space and at upsetting the classical image of the oriental Mediterranean Sea dominated by Genoa and Venice and their constants rivalries. The corpus gathered, composed of documents coming from the business practices - registers of accounts and correspondences - offer a lot of information on the trade which was then made in the Empire by the Florentine traders and allows to analyze their mechanisms of insertion on the most important markets of Ottoman Empire at the end of the $\mathrm{XV}^{\text {th }}$ century and at the beginning of the XVI ${ }^{\text {th }}$.

\section{Entrées d'index}

Mots-clés : Florence, Empire ottoman, produits textiles, marchands juifs, comptabilités, correspondances

Keywords : Florence - Ottoman Empire, textile products, Jewish merchants, account books, correspondences

\section{Notes de l'auteur}

Je tiens à remercier Mathieu Arnoux et Franco Franceschi pour leur lecture attentive, leurs suggestions et leurs conseils. 